\title{
Asymptotic simplicity and static data
}

\author{
Juan Antonio Valiente Kroon * \\ School of Mathematical Sciences, \\ Queen Mary, University of London, \\ Mile End Road, London E1 4NS, \\ United Kingdom.
}

June 11, 2018

\begin{abstract}
The present article considers time symmetric initial data sets for the vacuum Einstein field equations which in a neighbourhood of infinity have the same massless part as that of some static initial data set. It is shown that the solutions to the regular finite initial value problem at spatial infinity for this class of initial data sets extend smoothly through the critical sets where null infinity touches spatial infinity if and only if the initial data sets coincide with static data in a neighbourhood of infinity. This result highlights the special role played by static data among the class of initial data sets for the Einstein field equations whose development gives rise to a spacetime with a smooth conformal compactification at null infinity.
\end{abstract}

Keywords: General Relativity, asymptotic structure, spatial infinity

\section{Introduction}

The analysis of the structure of spatial infinity using the Einstein conformal field equations and the construction known as the cylinder at spatial infinity — see [8, 11, 19, 18, 20, 21, 22] — suggests that static initial data sets play a special role among the class of initial data sets for the vacuum Einstein field equations whose development has a smooth conformal compactification at null infinity. This issue is of fundamental importance in the construction of so-called asymptotically simple spacetimes. In connection with this expectation, the following theorem has been proved in 22]:

Theorem 1. Consider a time symmetric initial data set for the Einstein vacuum field equations which is conformally flat near infinity. The solutions to the regular finite initial value problem at spatial infinity is smooth through the critical sets where null infinity touches spatial infinity if and only if the data is exactly Schwarzschildean in a neighbourhood of infinity.

The context of this theorem is better appreciated if one recalls that the Schwarzschild spacetime is the only static spacetime with conformally flat slices - see 11. The use of conformally flat data sets in the analysis of $[22$ is a convenient technical assumption. Otherwise, the full complexity of time symmetric initial data sets would make it impossible to identify useful structures. The analysis in [22] builds upon the original analysis in [8] and the computer algebra calculations of [19, to make generic assertions about a certain type of asymptotic expansions for the conformal Einstein field equations made possible by the framework of the cylinder at spatial infinity.

The purpose of the present article is to consider an extension of Theorem 1 to the case of more general — non-conformally flat - time symmetric initial data sets. Again, explicit computer

\footnotetext{
*E-mail address: j.a.valiente-kroon@qmul.ac.uk
} 
algebra calculations carried out in 20] suggest the type of result that one can expect to prove. A fundamental difficulty in the way of constructing a generalisation to Theorem 1 is to obtain a parametrisation of time symmetric initial data sets for which it is simple to decide whether the data under consideration is static or not.

The properties of time symmetric initial data sets for the vacuum Einstein field equations, $\left(\tilde{\mathcal{S}}, \tilde{h}_{i j}\right)$, to be analysed in the present article are best discussed using a point compactification of the initial hypersurface $\mathcal{S}$ and an associated conformally rescaled metric $h_{i j}$ - the conformal metric. The 3 -manifold $\mathcal{S}$ contains singled out points $\left\{i_{1}, i_{2}, \ldots\right\}$ representing the points at infinity of the 3 -manifold $\tilde{\mathcal{S}}$. Our analysis will be local to one of these points, so it will be assumed, without loss of generality, that there is only one of them. This point will be deonted by $i$. In [6] it has been shown that static initial data sets satisfy a certain regularity condition involving the Cotton tensor and its higher order derivatives - see equation (6) in the main text. This property is, however, not enough to fully assert whether an initial data set is static - initial data sets like those of Misner [14] and Brill-Lindquist [3] satisfy the regularity condition as they are conformally flat, but clearly they do not, in general, give rise to static spacetimes. Further conditions need to be imposed on the initial data to obtain a static development. This gap between initial data sets satisfying the regularity condition and initial data sets which are exactly static at spatial infinity is still to be understood 1 .

The present article gets around the difficulty exposed in the previous paragraph by considering a restricted class of initial data sets for which it is simple to decide whether they are actually static or not. This class of initial data sets is constructed by looking at solutions to the equation giving rise to the conformal factor $\vartheta$ relating the 3 -metric $\tilde{h}_{i j}$ and $h_{i j}$, the Yamabe equation see equation (4) in the main text. In a suitably small neighbourhood of infinity, the solutions to the Yamabe equation can be split into its massless and massive parts. The massless part contains the information of the local geometry in a neighbourhood of $i$, whereas the massive part contains information of global nature - in particular the mass. The class of initial data sets to be used in the present article takes the solution of the Yamabe equation for static data and adds to it a further massive term that does not contribute to the mass so to obtain a new solution to the Yamabe equation - this can be done because of the linearity of the setting. This new solution to the Yamabe equation implies, in turn, a new solution to the constraint equations in a neighbourhood of infinity with the same conformal metric $\tilde{h}_{i j}$ as a static initial data set. We say that these solutions to the constraint equations have a static massless part. It can be verified that this class of initial data sets satisfies the regularity condition of [6] cfr. also equation (6) of the main text. This observation is of relevance in the present article as it has been shown in [8] that this condition is necessary for solutions to the regular finite initial value problem of the conformal Einstein field equations to extend smoothly through the critical sets where null infinity touches spatial infinity.

For the class of time symmetric initial data sets for the Einstein vacuum field equations discussed in the previous paragraph one can prove the following generalisation of Theorem 1 .

Theorem. Given an initial data set with static massless part, the solution to the regular finite initial value problem at spatial infinity is smooth through the critical sets if and only if the data is exactly static in a neighbourhood of infinity.

In other words, the smoothness of the development through the critical sets forces the extra massive part added to the (background) static initial data to vanish. A more precise version of this result will be given in the main text.

As in the case of the assumption of conformal flatness made in [22, the use of initial data sets with a static massless part is a useful technical assumption which allows to identify relevant structures in the conformal field equations. It is clear that not all time symmetric initial data sets admitting an analytic conformal compactification at infinity have a static massless part. A general version of the main theorem of this article can only be obtained once one knows what

\footnotetext{
${ }^{1}$ Recently, there has been some progress in this direction - H. Friedrich, parallel session talk in the GR19 Conference, Mexico.
} 
extra conditions have to be imposed on a generic time symmetric initial data set to have a static massless part — cfr. similar remarks in the previous paragraphs. This task requires learning how to exploit to the maximum extent the conformal gauge freedom implicit in the conformal Ansatz. As the conformal metric, $h_{i j}$, encodes all the freely specifiable information of a time symmetric initial data set, the extra requirements will have to be in the form of conformally invariant conditions on the conformal class.

The proof of the main theorem builds upon the analysis of the conformally flat case discussed in 22]. This analysis relied heavily on the use of computer algebra methods to transform the transport equations implied by the conformal field equations at the cylinder at spatial infinity into a system of reduced ordinary differential equations for which explicit solutions can be computed for any order of the expansion. The approach in the present article consists in conveniently grouping the various terms appearing in the transport equations in Schwarzschildean terms and deviations-from-Schwarzschild terms. The former are formally identical to terms appearing in [22] and thus, assertions about their smoothness can be readily given. It turns out that most of the terms that one needs to consider are Schwarzschildean terms. It is only in the last step of the argument that deviation terms arise. As it will be seen, their presence indicates that the extra massive part that has been added to the static data has to vanish up to a certain order - thus, putting into action an inductive argument from which the main theorem is obtained. Remarkably, essentially all the computer algebra required for this argument has already been performed in [22].

\section{Outline of the article}

Section 2 discusses some general properties of time symmetric solutions to the Einstein constraint equations in the conformal setting. It also introduces the class of time symmetric initial data sets that will be used in our subsequent analysis - initial data sets with a static massless part. Section 3 gives a concise summary of the framework of the cylinder at spatial infinity and of the so-called F-gauge. Its main purpose is to introduce the notation to be used in the rest of the article. It also provides an overview of the key properties of the transport equations implied by the conformal Einstein field equations at spatial infinity. Section 4 briefly discusses the key result of the construction of the cylinder at spatial infinity for static spacetimes - namely, that the structures are as smooth as they can be. Section 5 discusses key properties of initial data sets which are static up to a certain order - the results will be used extensively in the sequel. Section 6] contains the core of our analysis: a discussion of the properties of solutions to the transport equations at the cylinder at spatial infinity for data which is static up to a certain order. The results presented in this section take the form of an induction argument which leads, ultimately, to our main theorem in Section 7.

\section{Notation and conventions}

The present article is concerned with the asymptotic properties of spacetimes $\left(\tilde{\mathcal{M}}, \tilde{g}_{\mu \nu}\right)$ solving the Einstein vacuum field equations

$$
\tilde{R}_{\mu \nu}=0 \text {. }
$$

The metric $\tilde{g}_{\mu \nu}$ will be assumed to have signature $(+,-,-,-)$ and $\mu, \nu, \ldots$ are spacetime indices taking the values $0, \ldots, 3$. The spacetime $\left(\tilde{\mathcal{M}}, \tilde{g}_{\mu \nu}\right)$ will be thought of as the development of a time symmetric initial data set $\left(\tilde{\mathcal{S}}, \tilde{h}_{i j}\right)$ where $\tilde{\mathcal{S}}$ is an asymptotically Euclidean hypersurface. The metric $\tilde{h}_{i j}$ will be taken to have signature $(-,-,-)$. The indices $i, j, \ldots$ will be spatial ones taking the values $1,2,3$. The spinorial conventions of [15, 16] will be adopted. The present article draws heavily from the analysis in $[8,11,22$ so we have followed the notation and conventions of these references as closely as possible. 


\section{A class of time symmetric data}

For time symmetric initial data sets $\left(\tilde{\mathcal{S}}, \tilde{h}_{i j}\right)$ the Einstein vacuum field equations (1) imply the constraint equation

$$
\tilde{r}=0, \quad \text { on } \tilde{\mathcal{S}},
$$

where $\tilde{r}$ denotes the Ricci scalar of the metric $\tilde{h}_{i j}$.

Our analysis will be local to a neighbourhood of infinity. Hence, without loss of generality, only one asymptotically flat end will be assumed. The asymptotic flatness of the time symmetric initial data $\left(\tilde{\mathcal{S}}, \tilde{h}_{i j}\right)$ will be expressed in terms of conditions on a conformally rescaled manifold. For this, it will be assumed that there is a 3-dimensional, orientable, smooth compact manifold $\mathcal{S}$, a metric $h_{i j}$, a point $i \in \mathcal{S}$, a diffeomorphism $\Phi: \mathcal{S} \backslash\{i\} \rightarrow \tilde{\mathcal{S}}$ and a function $\Omega \in C^{2}(\mathcal{S}) \cap C^{\infty}(\mathcal{S} \backslash\{i\})$ with the properties

$$
\begin{aligned}
& \Omega(i)=0, \quad D_{j} \Omega(i)=0, \quad D_{j} D_{k} \Omega(i)=-2 h_{j k}(i), \\
& \Omega>0 \text { on } \mathcal{S} \backslash\{i\}, \\
& h_{i j}=\Omega^{2} \Phi_{*} \tilde{h}_{i j},
\end{aligned}
$$

where $D_{j}$ denotes the Levi-Civita covariant derivative of the 3 -metric $h_{i j}$. For the sake of simplicity the last condition will be written as $h_{i j}=\Omega^{2} \tilde{h}_{i j}$ so that $\mathcal{S} \backslash\{i\}$ is identified with $\tilde{\mathcal{S}}$. If assumptions (3a)-3c are satisfied, the pair $\left(\tilde{S}, \tilde{h}_{i j}\right)$ will be said to be asymptotically Euclidean and regular. Suitable punctured neighbourhoods of the point $i$ are mapped to the asymptotic end of $\tilde{\mathcal{S}}$.

\subsection{Asymptotically Euclidean and regular data}

The Hamiltonian constraint, equation (2), together with the boundary conditions (3a)-(3c) imply on $\mathcal{B}_{a}(i)$ the Yamabe Equation

$$
\left(\Delta_{h}-\frac{1}{8} r\right) \vartheta=-4 \pi \delta(i), \quad \vartheta \equiv \Omega^{-2}
$$

where $\delta(i)$ denotes the Dirac delta distribution with support on $i$ while $\Delta$ and $r$ correspond, respectively, to the Laplacian and the Ricci scalar of the conformal metric $h_{i j}$. For later use we define

$$
\omega=\frac{2 \Omega}{\sqrt{\left|D_{k} \Omega D^{k} \Omega\right|}} .
$$

It is well known - see e.g. [8, 11] - that if $a$ is suitably small, then the solutions to the Yamabe equation (4) on $\mathcal{B}_{a}(i)$ are of the form

$$
\vartheta=\frac{U}{|x|}+W, \quad|x|=\left(\left(x^{1}\right)^{2}+\left(x^{2}\right)^{2}+\left(x^{3}\right)^{2}\right)^{1 / 2}
$$

for some asymptotically Cartesian coordinates $x^{i}$. The terms $U /|x|$ and $W$ will be known, respectively, as the massless and massive parts of $\vartheta$. The function $U$, the Green function, satisfies the equation

$$
\left(\Delta_{h}-\frac{1}{8} r\right)\left(\frac{U}{|x|}\right)=-4 \pi \delta(i),
$$

and describes the local geometry in $\mathcal{B}_{a}(i)$. The function $W$ satisfies the equation

$$
\left(\Delta_{h}-\frac{1}{8} r\right) W=0
$$

annd contains information of global nature. In particular, $W(i)=m / 2$, where $m$ is the ADM mass of the initial data set. 
A rescaling

$$
h_{i j} \mapsto h_{i j}^{\prime}=\theta^{4} h_{i j}, \quad \Omega \mapsto \Omega^{\prime}=\theta^{2} \Omega,
$$

with a smooth positive factor $\theta$ satisfying $\theta(i)=1$ leaves the physical metric $\tilde{h}_{i j}=\Omega^{-2} h_{i j}$ unchanged. However, it implies the transitions

$$
\vartheta \mapsto \vartheta^{\prime}=\theta^{-1} \vartheta, \quad U \mapsto U^{\prime}=\frac{\left|x^{\prime}\right|}{|x|} \theta^{-1} U, \quad W \mapsto W^{\prime}=\theta^{-1} W,
$$

where $\left|x^{\prime}\right|$ is given in terms of the $h^{\prime}$-normal coordinates. There are several possibilities to fix this conformal gauge freedom. For the purpose of the present analysis it turns out that a good choice is that of the so-called conformal normal ( $c n$ )-gauge introduced in [8]. In the following definition let $l_{i j}$ denote the Schouten tensor of the metric $h_{i j}$.

Definition 1. The metric $h_{i j}$ is said to be in the cn-gauge if given a solution $(x(\lambda), b(\lambda))$ to the 3-dimensional conformal geodesic equations

$$
\begin{aligned}
& \dot{x}^{\nu} D_{\nu} \dot{x}^{\mu}=-2\left(b_{\nu} \dot{x}^{\nu}\right) \dot{x}^{\mu}+\left(h_{\lambda \rho} \dot{x}^{\lambda} \dot{x}^{\rho}\right) h^{\mu \nu} b_{\nu}, \\
& \dot{x}^{\nu} D_{\nu} b_{\mu}=\left(b_{\nu} \dot{x}^{\nu}\right) b_{\mu}-\frac{1}{2}\left(h^{\lambda \rho} b_{\lambda} b_{\rho}\right) h_{\mu \nu} \dot{x}^{\nu}+l_{\lambda \mu} \dot{x}^{\lambda},
\end{aligned}
$$

with initial conditions

$$
x(0)=i, \quad h(\dot{x}, \dot{x})(i)=-1, \quad b(0)=0,
$$

one has that

$$
\langle b, \dot{x}\rangle=0 .
$$

Remark. Let $h_{i j}$ be analytic in a neighbourhood of $i$. Assuming that $a$ is sufficiently small, there exists on $\mathcal{B}_{a}(i)$ a unique analytic rescaling $h_{i j} \mapsto h_{i j}^{\prime}=\theta^{4} h_{i j}$ for which $h_{i j}^{\prime}$ is in the cn-gauge. The metric and connection remain unchanged at $i$. As it will be discussed in the sequel, the practical advantage of the cn-gauge is that it renders simpler multipolar expansions for various quantities of interest.

\subsection{Asymptotically static data}

Let $\left(\mathcal{S}, \stackrel{\circ}{h}_{i j}\right)$ denote a static initial data set given in the cn-gauge, and let

$$
\grave{\vartheta} \equiv \frac{\stackrel{\circ}{U}}{|x|}+\stackrel{\circ}{W}
$$

denote the corresponding solution to the Yamabe equation (4) in a suitably small neighbourhood $\mathcal{B}_{a}(i)$. The static initial data set can be specified entirely in terms of its multipole moments - see e.g. [12]. This important fact will, however, not be exploited here. As a consequence of the analysis in [11, 12] one has the following:

Proposition 1. Let $\left(\tilde{\mathcal{S}}, \tilde{h}_{i j}\right)$ be a static initial data set and let $\mathcal{B}_{a}(i)$ be a suitably small neighbourhood of $i$. If the conformal metric $\stackrel{\circ}{h}_{i j}$ satisfies the cn-gauge $\mathcal{B}_{a}(i)$, then there exist normal coordinates $x^{i}$ such that $\stackrel{\circ}{h}_{i j}, \stackrel{\circ}{U}$ and $\stackrel{\circ}{W}$ are analytic in the neighbourhood.

One also has that $-\mathrm{cfr}$. [2, 8 -

Proposition 2. The Cotton-Bach tensor $\stackrel{\circ}{i j}_{i j}$ of the conformal metric $\stackrel{\circ}{h}_{i j}$ of a static initial data set $\left(\tilde{\mathcal{S}}, \tilde{h}_{i j}\right)$ satisfies the regularity condition

$$
\mathcal{C}\left(D_{k_{q}} \cdots D_{k_{1}} \stackrel{\circ}{b l}_{k l}\right)(i)=0, \quad q=0,1, \ldots
$$

Remark 1. Given a sequence of multipoles for a static solution, one can always assume without loss of generality, that the dipolar terms vanish - this amounts to the gauge choice of working in the centre of mass. It can be readily verified that this assumption has the consequence that static data in the cn-gauge satisfies

$$
\stackrel{\circ}{W}=\frac{m}{2}+\mathcal{O}\left(|x|^{2}\right) .
$$


The latter form of the function $\stackrel{\circ}{W}$ will be assumed in the sequel.

Remark 2. If $\stackrel{\circ}{U}=1$ and $\stackrel{\circ}{W}=m / 2$ in $\mathcal{B}_{a}(i)$, then the static initial data set corresponds to initial data for the Schwarzschild spacetime.

The following observation will be crucial in our subsequent analysis. Let $\breve{W}$ satisfy

$$
\left(\Delta_{h}-\frac{1}{8} r\right) \breve{W}=0, \quad \breve{W}(i)=0 .
$$

Clearly, due to linearity one has that

$$
\vartheta=\frac{\stackrel{\circ}{|x|}}{|x|} \stackrel{\circ}{W}+\breve{W}
$$

is also a solution to the Yamabe equation (4) with the same boundary conditions in $\mathcal{B}_{a}(i)$. Due to the analyticity of $\stackrel{\circ}{h}_{i j}$, the function $\breve{W}$ will also be analytic. Time symmetric initial data sets, $\left(\tilde{\mathcal{S}}, \tilde{h}_{i j}\right)$, constructed out of the conformal metric $\stackrel{\circ}{h}_{i j}$ and the conformal factor $\vartheta$ given by equation (17) will be said to have a static massless part. Moreover, following the ideas in [22], we make the following ad hoc definition:

Definition 2. A time symmetric initial data set in the cn-gauge will be said to be static up to order $p_{\bullet}$ if and only if there exists coordinates $x^{i}$ in a suitable neighbourhood $\mathcal{B}_{a}(i)$ of infinity such that the solution $\vartheta$ to the Yamabe equation (4) is of the form

$$
\vartheta=\frac{\stackrel{\circ}{\mid}}{|x|}+\stackrel{\circ}{W}+\breve{W}, \quad \breve{W}=\sum_{p=p \bullet+1}^{\infty} \breve{w}_{i_{1} \cdots i_{p}} x^{i_{1}} \cdots x^{i_{p}},
$$

where $\stackrel{\circ}{U} /|x|$ and $\stackrel{\circ}{W}$ are, respectively, the massless and massive parts of a static initial data set.

Remark. It can be shown that in the cn-gauge the polynomials

$$
\breve{w}_{i_{1} \cdots i_{p_{\bullet}+1}} x^{i_{1}} \cdots x^{i_{i_{\bullet}+1}}, \quad \breve{w}_{i_{1} \cdots i_{p_{\bullet}+2}} x^{i_{1}} \cdots x^{i_{p_{\bullet}+2}}, \quad \breve{w}_{i_{1} \cdots i_{p_{\bullet}+3}} x^{i_{1}} \cdots x^{i_{p_{\bullet}+3}}
$$

are harmonic with respect to the flat Laplacian. That is, the constant tensors

$$
\breve{w}_{i_{1} \cdots i_{p_{\bullet}+1}}, \quad \breve{w}_{i_{1} \cdots i_{p_{\bullet}+2}}, \quad \breve{w}_{i_{1} \cdots i_{p_{\bullet}+3}}
$$

are trace-free with respect to the flat metric $\delta_{i j}$.

\subsection{Properties of data which is static up to a certain order}

Consistent with Definition 2, we write

$$
\Omega=\stackrel{\Omega}{\Omega} \breve{\Omega}, \quad \omega=\dot{\omega}+\breve{\omega},
$$

where $\stackrel{\Omega}{\Omega}$ and $\stackrel{\circ}{\omega}$ are, respectively, the static parts of $\Omega$ and $\omega$ (obtained by setting $\breve{W}=0$ ). Their non-static parts $\breve{\Omega}$ and $\breve{\omega}$ are then obtained via

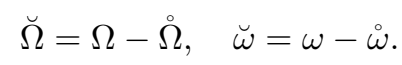

The following result is obtained by direct computation observing expression (7):

Lemma 1. Let $\left(\tilde{\mathcal{S}}, \tilde{h}_{i j}\right)$ be an initial data set which is static up to order $p_{\bullet} \geq 2$. Then

$$
\breve{\Omega}=\mathcal{O}\left(|x|^{p \bullet+4}\right), \quad \breve{\omega}=\mathcal{O}\left(|x|^{p \bullet+3}\right) .
$$

Let $b_{i j}$ denote the Cotton-Bach tensor of the conformal metric $h_{i j}$ of an initial data set which is static up to a certain order in the sense of Definition 2. Crucially, one has that: 
Lemma 2. Let $\left(\tilde{\mathcal{S}}, \tilde{h}_{i j}\right)$ be an initial data set which is static up to order $p \bullet \geq 2$. Then

$$
\mathcal{C}\left(D_{i_{q}} \cdots D_{i_{1}} b_{i j}\right)(i)=0, \quad q=0, \ldots, p_{\bullet},
$$

where $\mathcal{C}$ denotes the operation of taking the symmetric trace-free part.

Proof. As already discussed, Proposition 2 shows that the property holds for exactly static initial data sets. In 8 it has been shown that this property concerns only the massless part of time symmetric data sets. The result follows then by observing that an initial data set which is static up to a certain order has the same massless part as the one of a static initial data set so that $b_{i j}=\stackrel{\circ}{i j}_{i j}$.

\section{The cylinder at spatial infinity}

In 8] a representation of the region of spacetime close to null infinity and spatial infinity has been introduced - see also the comprehensive discussion in [11. The standard representation of this region of spacetime depicts $i^{0}$ as a point. In contrast, the representation introduced in 8 depicts spatial infinity as a cylinder - the cylinder at spatial infinity. This construction is briefly reviewed for the case of time symmetric initial data sets with an analytic conformal metric in a neighbourhood $\mathcal{B}_{a}(i)$ of infinity. The reader is referred to [8, 11] for a thorough discussion of the details - see also [22].

\subsection{The Manifold $\mathcal{C}_{a}$}

Starting from the initial hypersurface $\mathcal{S}$, the construction introduced in 8 makes use of a blowup of the point $i \in \mathcal{S}$ to the 2 -sphere $\mathbb{S}^{2}$. This blow-up requires the introduction of a particular bundle of spin-frames over $\mathcal{B}_{a}$. Consider the (conformally rescaled) spacetime $\left(\mathcal{M}, g_{\mu \nu}\right)$ obtained as the development of the time symmetric initial data set $\left(\mathcal{S}, h_{i j}\right)$. Let $S L(\mathcal{S})$ be the set of spin dyads $\delta=\left\{\delta_{A}\right\}_{A=0,1}$ on $\mathcal{S}$ which are normalised with respect to the alternating spinor $\epsilon_{A B}$ in such a way that $\epsilon_{01}=1$. Let $\tau=\sqrt{2} e_{0}$, where $e_{0}$ is the future $g$-unit normal of $\mathcal{S}$ and $\tau_{A A^{\prime}}$ its spinorial counterpart. The spinor $\tau_{A A^{\prime}}$ enables the introduction of space-spinors - sometimes also called $S U(2)$ spinors, see [1, 4, 17. It defines a sub-bundle $S U(\mathcal{S})$ of $S L(\mathcal{S})$ with structure group $S U(2, \mathbb{C})$ and projection $\pi$ onto $\mathcal{S}$. Given a spinorial dyad $\delta \in S U(\mathcal{S})$ one can define an associated vector frame $e_{a}, a=1,2,3$. We shall restrict our attention to dyads related to frames $\left\{e_{j}\right\}_{j=0, \cdots, 3}$ on $\mathcal{B}_{a}$ such that $e_{3}$ is tangent to the $h$-geodesics starting at $i$. Let $\check{H}$ denote the horizontal vector field on $S U(\mathcal{S})$ projecting to the radial vector $e_{3}$. The fibre $\pi^{-1}(i) \subset S U(\mathcal{S})$ (the fibre "over" $i$ ) can be parametrised by choosing a fixed dyad $\delta^{*}$ and then letting the group $S U(2, \mathbb{C})$ act on it. Let $(-a, a) \ni \rho \mapsto \delta\left(\rho, t^{A}{ }_{B}\right) \in S U(\mathcal{S})$ be the integral curve to the vector $\tilde{H}$ satisfying $\delta\left(0, t_{B}^{A}\right)=\delta\left(t_{B}^{A}\right) \in \pi^{-1}(i)$. With this notation one defines the set

$$
\mathcal{C}_{a}=\left\{\delta\left(\rho, t^{A}{ }_{B}\right) \in S U\left(\mathcal{B}_{a}\right)|| \rho \mid<a, t^{A}{ }_{B} \in S U(2, \mathbb{C})\right\},
$$

which is a smooth submanifold of $S U(\mathcal{S})$ diffeomorphic to $(-a, a) \times S U(2, \mathbb{C})$. It follows that the projection map $\pi$ of the bundle $S U(\mathcal{S})$ maps $\mathcal{C}_{a}$ into $\mathcal{B}_{a}$. The manifold $\mathcal{C}_{a}$ inherits a number of structures from $\mathcal{B}_{a}$. In particular, the solder and connection forms can be pulled back to smooth 1 -forms on $\mathcal{C}_{a}$ satisfying the structure equations which relate them to the curvature form. The explicit form of the structure equations will not be required here. As $\mathcal{S}$ is 3-dimensional, the full content of its curvature can be expressed in terms of the spinorial counterpart of the trace-free Ricci tensor $s_{A B C D}$ and the Ricci scalar $r$.

In the sequel $t_{B}^{A} \in S U(2, \mathbb{C})$ and $\rho \in \mathbb{R}$ will be used as coordinates on $\mathcal{C}_{a}$. Consequently, one has that $\check{H}=\partial_{\rho}$. Vector fields $X_{ \pm}, X$ relative to the $S U(2, \mathbb{C})$-dependent part of the coordinates can be introduced by requiring the commutation relations

$$
\left[X, X_{+}\right]=2 X_{+}, \quad\left[X, X_{-}\right]=-2 X_{-}, \quad\left[X_{+}, X_{-}\right]=-X,
$$

and by requiring that they commute with $\check{H}=\partial_{\rho}$. More importantly, it can be seen that for $p \in \mathcal{B}_{a} \backslash\{i\}$ the projections of the fields $\partial_{\rho}, X_{ \pm}$span the tangent space at $p$. Given these vector 
fields, define the frame $c_{A B}=c_{(A B)}$ by

$$
c_{A B}=c_{A B}^{1} \partial_{\rho}+c_{A B}^{+} X_{+}+c_{A B}^{-} X_{-},
$$

where

$$
c_{A B}^{1}=x_{A B}, \quad c_{A B}^{+}=\frac{1}{\rho} z_{A B}+\check{c}_{A B}^{+}, \quad c_{A B}^{-}=\frac{1}{\rho} y_{A B}+\check{c}_{A B}^{-},
$$

with constant spinors $x_{A B}, y_{A B}$ and $z_{A B}$ given by

$$
x_{A B} \equiv \sqrt{2} \epsilon_{(A}{ }^{0} \epsilon_{B)}{ }^{1}, \quad y_{A B} \equiv-\frac{1}{\sqrt{2}} \epsilon_{A}{ }^{1} \epsilon_{B}{ }^{1}, \quad z_{A B}=\frac{1}{\sqrt{2}} \epsilon_{A}{ }^{0} \epsilon_{B}{ }^{0},
$$

and analytic spinor fields satisfying

$$
\check{c}_{A B}^{ \pm}=\mathcal{O}(\rho), \quad \check{c}_{01}^{ \pm}=0 .
$$

For the frame $c_{A B}$, the connection coefficients, $\gamma_{A B C D}$, are given by

$$
\gamma_{A B C D}=\frac{1}{\rho} \gamma_{A B C D}^{*}+\check{\gamma}_{A B C D}, \quad \gamma_{A B C D}^{*}=\frac{1}{2}\left(\epsilon_{A C} x_{B D}+\epsilon_{B D} x_{A C}\right),
$$

where

$$
\check{\gamma}_{A B C D}=\mathcal{O}(\rho), \quad \check{\gamma}_{11 C D}=0 .
$$

\subsection{An orthonormal basis for functions on $S U(2, \mathbb{C})$}

Given $t^{A}{ }_{B} \in S U(2, \mathbb{C})$, define

$$
\begin{aligned}
& T_{m}{ }_{k}^{j}\left(t^{A}{ }_{B}\right)=\left(\begin{array}{c}
m \\
j
\end{array}\right)^{1 / 2}\left(\begin{array}{c}
m \\
k
\end{array}\right)^{1 / 2} t_{\left(A_{1}\right.} \cdots t^{\left.B_{m}\right)_{j}}{ }_{\left.A_{m}\right)_{k}}, \\
& T_{0}{ }_{0}^{0}\left(t^{A}{ }_{B}\right)=1,
\end{aligned}
$$

with $j, k=0, \ldots, m$ and $m=1,2,3, \ldots$ The subindex expression $\left(A_{1} \cdots A_{m}\right)_{k}$ means that the indices are symmetrised and then $k$ of them are set equal to 1 , while the remaining ones are set to 0 . Details about the properties of these functions can be found in [5, 8. The functions $\sqrt{m+1} T_{m k}{ }_{k}^{j}$ form a complete orthonormal set in the Hilbert space $L^{2}(\mu, S U(2, \mathbb{C}))$, where $\mu$ denotes the normalised Haar measure on $S U(2, \mathbb{C})$. The action of the differential operators $X_{ \pm}$ on the functions $T_{m}{ }^{k}$ is given by

$$
X_{+} T_{m}{ }_{j}^{k}=\sqrt{j(m-j+1)} T_{m}{ }_{j-1}^{k}, \quad X_{-} T_{m}{ }_{j}^{k}=-\sqrt{(j+1)(m-j)} T_{m}{ }_{j+1}^{k} .
$$

In the sequel, we will need to linearise products of the form $T_{m}{ }_{k}^{i}$. To this end, we use the formula:

$$
\begin{gathered}
T_{i_{1} k_{1}}^{j_{1}} \times T_{i_{2}}{ }^{j_{2}} k_{2}=\sum_{p=0}^{\mu} D\left(i_{1}, j_{1}, k_{1} ; i_{2}, j_{2}, k_{2} ; i_{1}+i_{2}-2 p, j_{1}+j_{2}-p, k_{1}+k_{2}-p\right) \\
\times T_{i_{1}+i_{2}-2 p}{ }^{j_{1}+j_{2}-p}{ }_{k_{1}+k_{2}-p},
\end{gathered}
$$

with $\mu=\min \left\{i_{1}, i_{2}, j_{1}+j_{2}, k_{1}+k_{2}\right\}$ and

$$
D\left(i_{1}, j_{1}, k_{1} ; i_{2}, j_{2}, k_{2} ; l, m, n\right)=C\left(i_{1}, j_{1} ; i_{2}, j_{2} ; l, m\right) \overline{C\left(i_{1}, k_{1} ; i_{2}, k_{2} ; l, m\right)},
$$

and $C(i, j ; k, l ; m, n)$ the Clebsch-Gordan coefficients of $S U(2, \mathbb{C})$. 


\subsection{Normal expansions at $\mathcal{I}^{0}$}

In the sequel, we will consider the lift of analytic fields defined on $\mathcal{B}_{a}$ to $\mathcal{C}_{a}$. In particular, the lift of $|x|$ is $\rho$. More generally, let $\xi_{A_{1} B_{1} \cdots A_{l} B_{l}}$ denote a spinorial field on $\mathcal{B}_{a}$. Denote, again, by $\xi_{A_{1} B_{1} \cdots A_{l} B_{l}}$ its lift to $\mathcal{C}_{a}$. Denote by $\xi_{j}=\xi_{\left(A_{1} B_{1} \cdots A_{l} B_{l}\right)_{j}}, 0 \leq j \leq l$ its essential components. The function $\xi_{j}$ has spin weight $s=l-j$ and a unique expansion of the form

$$
\xi_{j}=\sum_{p=0}^{\infty} \xi_{j, p} \rho^{p}, \quad \xi_{j, p}=\sum_{q=\max \{|l-j|, l-p\}}^{p+l} \sum_{k=0}^{2 q} \xi_{j, p ; 2 q, k} T_{2 q}{ }^{k}{ }^{2 q-l+j},
$$

with complex coefficients $\xi_{j, p ; 2 q, k}$. More generally, we shall consider symmetric spinorial fields $\xi_{A_{1} \cdots A_{r}}$ on $\mathcal{C}_{a}$ with independent components $\xi_{j}=\xi_{\left(A_{1} \cdots A_{2 r}\right)_{j}}, 0 \leq j \leq 2 r$, and spin-weight $s=r-j$ which do not descend to analytic spinor fields on $\mathcal{B}_{a}$. In this case one has that

$$
\xi_{j}=\sum_{p=0}^{\infty} \xi_{j, p} \rho^{p}, \quad \xi_{j, p}=\sum_{q=|r-j|}^{q(p)} \sum_{k=0}^{2 q} \xi_{j, p ; 2 q, k} T_{2 q}{ }^{k} q-r+j,
$$

where one has a priori that $0 \leq|r-j| \leq q(p)$. An expansion of the latter form will be said to be of type $q(p)$.

\subsubsection{Particular results concerning expansion types}

We recall the following result in [8]:

Lemma 3. Assuming the cn-gauge and if for some non-negative integer $k$ it holds

$$
D_{\left(E_{p} F_{p}\right.} \cdots D_{E_{1} F_{1}} b_{A B C D)}(i)=0, \quad p=0,1, \cdots, k
$$

then we have the following expansion types:

$$
\begin{aligned}
& \operatorname{type}(r)=\operatorname{type}(W)=p, \\
& \operatorname{type}\left(s_{A B C D}\right)=p, \\
& \operatorname{type}\left(\check{\gamma}_{A B C D}\right)=p-1, \\
& \operatorname{type}\left(\check{c}_{A B}^{ \pm}\right)=p-1, \\
& \operatorname{type}(U-1)=p-2 .
\end{aligned}
$$

In addition, we have that

$$
U=1+O\left(\rho^{4}\right), \quad s_{A B C D}=\mathcal{O}\left(\rho^{2}\right), \quad \check{c}_{A B}^{ \pm}=\mathcal{O}\left(\rho^{3}\right), \quad \check{\gamma}_{A B C D}=\mathcal{O}\left(\rho^{3}\right) .
$$

Remark. The particular structure of the expansions dictated by this lemma greatly simplifies the subsequent analysis.

Important for our subsequent analysis is the particular form of the lift to $\mathcal{C}_{a}$ of the function $\breve{W}$ appearing in the conformal factor $\vartheta$ for data which is static up to order $p_{\bullet}$. Using the methods of [8] one finds that

$$
\breve{W}=\sum_{p=p \bullet+1}^{p \bullet+3} \sum_{k=0}^{2 p} \frac{1}{p !} \breve{w}_{p ; 2 p, k} T_{2 p}{ }^{k}{ }_{p} \rho^{p}+\sum_{p=p \bullet+4}^{\infty} \sum_{q=0}^{p} \sum_{k=0}^{2 q} \frac{1}{p !} \breve{w}_{p ; 2 q, k} T_{2 q}{ }^{k}{ }_{q} \rho^{p},
$$

where the coefficients $\breve{w}_{p ; 2 q, k}$ are related to the constant tensors $w_{i_{1} \cdots i_{p}}$ via contractions with Infeld-van der Waerden symbols. In particular, it can be seen that

$$
\breve{w}_{p ; 2 q, k}=0, \quad q=0, \ldots p, \quad k=0, \ldots 2 q \Longleftrightarrow w_{i_{1} \ldots i_{p}}=0
$$

The key observation in equation (10) is that the terms of order $\mathcal{O}\left(\rho^{p \bullet+1}\right), \mathcal{O}\left(\rho^{p \bullet+2}\right), \mathcal{O}\left(\rho^{p \bullet+3}\right)$ are formally identical to the ones appearing in the corresponding expansions for the function $\breve{W}$ of data which is Schwarzschildean up to order $p_{\bullet}(\stackrel{\circ}{W}=m / 2)$. In other words,

$$
\begin{aligned}
& \breve{w}_{p_{\bullet}+1 ; q, k}=0, \quad q=0, \ldots p_{\bullet}+1, \quad k=0, \ldots q, \\
& \breve{w}_{p \bullet+2 ; q, k}=0, \quad q=0, \ldots p_{\bullet}+2, \quad k=0, \ldots q, \\
& \breve{w}_{p_{\bullet}+3 ; q, k}=0, \quad q=0, \ldots p_{\bullet}+3, \quad k=0, \ldots q .
\end{aligned}
$$




\subsection{The spacetime Friedrich gauge}

The formulation of the initial value problem near spatial infinity presented in 8 employs gauge conditions based on timelike conformal geodesics. The conformal geodesics are curves which are autoparallel with respect to a Weyl connection - i.e. a torsion-free connection which is not necessarily the Levi-Civita connection of a metric. An analysis of Weyl connections in the context of the conformal field equations has been given in [7. In terms of this gauge based on conformal geodesics — which shall be called the Friedrich gauge or F-gauge for short - the conformal factor of the spacetime can be determined explicitly in terms of the initial data for the Einstein vacuum equations. Hence, provided that the congruence of conformal geodesics and the fields describing the gravitational field extend in a regular manner to null infinity, one has complete control over the location of null infinity. This can be ensured by making $\mathcal{B}_{a}$ suitably small. In addition, the F-gauge renders a particularly simple representation of the propagation equations. Using this framework, the singular initial value problem at spatial infinity can be reformulated into another problem where null infinity is represented by an explicitly known hypersurface and where the data are regular at spacelike infinity. The construction of the bundle manifold $\mathcal{C}_{a}$ and the blowing up of the point $i \in \mathcal{B}_{a}$ to the set $\mathcal{I}^{0} \subset \mathcal{C}_{a}$, briefly described in section 3.1 are the first steps in the construction of this regular setting. The next step is to introduce a rescaling of the frame bundle so that fields that are singular at $\mathcal{I}^{0}$ become regular.

Following the discussion of [8] assume that given the development of data prescribed on $\mathcal{B}_{a}$, the timelike spinor $\tau^{A A^{\prime}}$ introduced in section 3.1 is tangent to a congruence of timelike conformal geodesics which are orthogonal to $\mathcal{B}_{a}$. The canonical conformal factor rendered by this congruence of conformal geodesics is given in terms of an affine parameter $\tau$ of the conformal geodesics by

$$
\Theta=\kappa^{-1} \Omega\left(1-\frac{\kappa^{2} \tau^{2}}{\omega^{2}}\right)
$$

with $\omega$ as given by equation (5) and where $\Omega=\vartheta^{-2}$ and $\vartheta$ solves the Yamabe equation (4) - see [7, 8, 10]. The function $\kappa>0$ expresses the remaining conformal freedom in the construction. It will be taken to be of the form $\kappa=\kappa^{\prime} \rho$, with $\kappa^{\prime}$ analytic, $\kappa^{\prime}(i)=1$. Associated to the conformal factor $\Theta$ there is a 1 -form $d_{\mu}$ from which the Weyl connection can be obtained. In spinorial terms, one has that for conformally flat data

$$
d_{A A^{\prime}}=\frac{1}{\sqrt{2}} \tau_{A A^{\prime}} \partial_{\tau} \Theta-\tau_{A^{\prime}}{ }^{\prime} d_{A B}, \quad d_{A B}=2 \rho\left(\frac{U x_{A B}-\rho D_{A B} U-\rho^{2} D_{A B} W}{(U+\rho W)^{3}}\right) .
$$

The function $\kappa$ in the conformal factor $\Theta$, induces a scaling $\delta_{A} \mapsto \kappa^{1 / 2} \delta_{A}$ of the spin frame. Accordingly, one considers the bundle manifold $\mathcal{C}_{a, \kappa}=\kappa^{1 / 2} \mathcal{C}_{a}$ of scaled spinor frames. Using $\mathcal{C}_{a, \kappa}$ one defines the set

$$
\mathcal{M}_{a, \kappa}=\left\{(\tau, q) \mid q \in \mathcal{C}_{a, \kappa},-\frac{\omega(q)}{\kappa(q)} \leq \tau \leq \frac{\omega(q)}{\kappa(q)}\right\}
$$

which, assuming that the congruence of null geodesics and the relevant fields extend adequately, can be identified with the development of $\mathcal{B}_{a}$ up to null infinity - that is, the region of spacetime near null and spatial infinity. In addition, one defines the sets:

$$
\begin{aligned}
& \mathcal{I}=\left\{(\tau, q) \in \mathcal{M}_{a, \kappa}|\rho(q)=0,| \tau \mid<1\right\}, \\
& \mathcal{I}^{ \pm}=\left\{(\tau, q) \in \mathcal{M}_{a, \kappa} \mid \rho(q)=0, \tau= \pm 1\right\}, \\
& \mathscr{I}^{ \pm}=\left\{(\tau, q) \in \mathcal{M}_{a, \kappa} \mid \rho(q)>0, \quad \tau= \pm \frac{\omega(q)}{\kappa(q)}\right\},
\end{aligned}
$$

which will be referred to as, respectively, the cylinder at spatial infinity, the critical sets and future and past null infinity. In order to coordinatise the hypersurfaces of constant parameter $\tau$, one extends the coordinates $\left(\rho, t^{A}{ }_{B}\right)$ off $\mathcal{C}_{a, \kappa}$ by requiring them to be constant along the conformal geodesics - i.e. one has a system of conformal Gaussian coordinates. 
Remark. For the purpose of the analysis carried out in this article it turns out that the most convenient choice of the function $\kappa$ in the conformal factor $\Theta$ of equation (11) is

$$
\kappa=\rho .
$$

This leads to considerable simplifications in all the relevant expressions. From this point onwards, this choice will always be assumed.

\subsection{The evolution equations}

On the manifold $\mathcal{M}_{a, \kappa}$ it is possible to introduce a calculus based on the derivatives $\partial_{\tau}$ and $\partial_{\rho}$ and on the operators $X_{+}, X_{-}$and $X$. The operators $\partial_{\rho}, X_{+}, X_{-}$and $X$ originally defined on $\mathcal{C}_{a}$ can be suitably extended to the rest of the manifold by requiring them to commute with the vector field $\partial_{\tau}$. In order to derive the propagation equations, a frame $c_{A A^{\prime}}$ and the associated spin connection coefficients $\Gamma_{A A^{\prime} B C}$ of the Weyl connection $\nabla$ will be used. The gravitational field is, in addition, described by the spinorial counterparts of the Schouten tensor of the Weyl connection, $\Theta_{A A^{\prime} B B^{\prime}}$, and of the rescaled Weyl tensor, $\phi_{A B C D}$ - see [7, 8, 11. Let $\phi_{i} \equiv \phi_{(A B C D)_{i}}$. In the present gauge, the information of the spacetime spinors $c_{A A^{\prime}}^{\mu}, \Theta_{A A^{\prime} B B^{\prime}}$ and $\Gamma_{A A^{\prime} B C}$ is encoded, respectively, in space spinors $c_{A B}^{\mu}, \Theta_{A B C D}$ and $\Gamma_{A B C D}$ - see [1] for the detailed relation between the two sets of spinors.

In what follows, we will arrange the independent components of the spinorial fields $c_{A B}^{\mu}$, $\Theta_{A B C D}$ and $\Gamma_{A B C D}$ in the vector $\boldsymbol{v}$, and those of $\phi_{A B C D}$ in the vector $\boldsymbol{\phi}$. Suitable field equations for the fields contained in $\boldsymbol{v}$ and $\phi$ can be obtained from the first and second Cartan structure equations, the Bianchi identity of the conformally rescaled spacetime $\left(\mathcal{M}, g_{\mu \nu}\right)$, and the Bianchi identity of the physical spacetime $\left(\tilde{\mathcal{M}}, \tilde{g}_{\mu \nu}\right)$ - see e.g. [9, 11] for details. A solution to the equations thus constructed implies a solution to the vacuum Einstein field equations. The vector $\boldsymbol{v}$ has 45 independent complex components, while $\phi$ has 5 independent complex components. Using the F-gauge it can be shown that the extended conformal field equations given in [8] imply the following evolution equations for the unknowns $\boldsymbol{v}$

$$
\partial_{\tau} \boldsymbol{v}=\mathbf{K} \cdot \boldsymbol{v}+\mathbf{Q}(\boldsymbol{v}, \boldsymbol{v})+\mathbf{L} \cdot \boldsymbol{\phi},
$$

where $\mathbf{K}$ and $\mathbf{Q}$ denote, respectively, linear and quadratic constant matrix-valued functions with constant entries, and $\mathbf{L}$ is a linear matrix-valued function with coefficients depending on the coordinates and such that $\left.\mathbf{L}\right|_{\rho=0}=0$. For the unknowns $\phi$, the Bianchi identity $\nabla^{A A^{\prime}} \phi_{A B C D}=0$ implies, respectively, a set of propagation and constraint equations of the form:

$$
\begin{aligned}
& \mathbf{E} \cdot \partial_{\tau} \boldsymbol{\phi}+\mathbf{A}^{\mu}(\mathbf{c}) \partial_{\mu} \boldsymbol{\phi}=\mathbf{F}(\boldsymbol{\Gamma}) \cdot \boldsymbol{\phi}, \\
& \mathbf{B}^{\mu}(\mathbf{c}) \cdot \partial_{\mu} \boldsymbol{\phi}=\mathbf{G}(\boldsymbol{\Gamma}),
\end{aligned}
$$

where $\mathbf{E}$ denotes the $5 \times 5$ unit matrix, $\mathbf{A}^{\mu}(\mathbf{c})$ and $\mathbf{B}^{\mu}(\mathbf{c}), \mu=0, \ldots, 3$, are, respectively, $5 \times 5$ and $3 \times 5$ constant matrix-valued linear functions depending on the frame field coefficients $c_{A B}^{\mu}$. Finally, $\mathbf{F}(\boldsymbol{\Gamma})$ and $\mathbf{G}(\boldsymbol{\Gamma})$ denote constant matrix-valued linear functions of the connection coefficients $\Gamma_{A B C D}$.

\subsection{Initial data for the evolution equations}

For quick reference, we include here the expressions for the initial data for the curvature components appearing in equations (14)-(15b). These expressions can be deduced from the conformal constraint equations - see [8]. On $\mathcal{C}_{a, \kappa}$ one has that:

$$
\begin{aligned}
& \Theta_{A B C D}=-\frac{\kappa^{2}}{\Omega} D_{(A B} D_{C D)} \Omega+\frac{1}{12} \kappa^{2} r h_{A B C D} \\
& \phi_{A B C D}=\frac{\kappa^{3}}{\Omega^{2}}\left(D_{(A B} D_{C D)}+\Omega s_{A B C D}\right)
\end{aligned}
$$

where, $\Omega$ denotes the lift to $\mathcal{C}_{a, \kappa}$ of the conformal factor obtained from equation (77). The expressions for the frame and connection coefficients do not involve the function $\Omega$, and thus their detailed expressions will not be required here. 


\subsection{The transport equations}

Consider now the system (14)-15a) with data given on $\mathcal{C}_{a, \kappa}$, and let be given a neighbourhood $\mathcal{W}$ of $\mathcal{C}_{a, \kappa}$ in $\mathcal{M}_{a, \kappa}$ on which a unique smooth solution of the Cauchy problem exists. From the point of view of the propagation equations, the subset $\mathcal{W} \cap \mathcal{I}$ is a regular hypersurface. Introduce the notation

$$
\left.\boldsymbol{v}^{(0)} \equiv \boldsymbol{v}\right|_{\mathcal{W} \cap \mathcal{I}},\left.\quad \phi^{(0)} \equiv \phi\right|_{\mathcal{W} \cap \mathcal{I}}
$$

Due to the property $\left.\mathbf{L}\right|_{\rho=0}=0$, equations (14) decouple from equations (15a) and can be integrated on $\mathcal{W} \cap \mathcal{I}$ using the observation that the restriction of the initial data to $\mathcal{I}^{0}$ coincides with Minkowski data. The solutions thus obtained extend analytically to the whole of $\mathcal{I}$ and in particular to the critical sets $\mathcal{I}^{ \pm}$. The set $\mathcal{I}$ turns out to be a total characteristic of the system (14)-(15a) in the sense that the whole system reduces to an interior system on $\mathcal{I}$. Moreover, the constraint equations (15b) also reduce to an interior system on $\mathcal{I}$. As mentioned before, this feature is a consequence of the fact that the unphysical metric $g_{\mu \nu}$ determined by a solution to the conformal field equations degenerates as $\rho \rightarrow 0$.

A crucial structural property is that

$$
\mathbf{E}+\mathbf{A}^{0}(\mathbf{c})=\operatorname{diag}(1+\tau, 1,1,1,1-\tau) \quad \text { on } \mathcal{I},
$$

so that the matrix $\mathbf{E}+\mathbf{A}^{0}(\mathbf{c})$ which is positive definite for $|\tau|<1$ degenerates at $\mathcal{I}^{ \pm}$. Understanding the effects of this degeneracy is the main motivation behind the analysis in the present article and that in 22 .

In order to analyse the effects of the degeneracy of the matrix $\mathbf{E}+\mathbf{A}^{0}(\mathbf{c})$ we will consider the hierarchy of transport equations which is obtained by repeated application of the operator $\partial_{\rho}$ to equations (14)-(15b) and then evaluation on $\mathcal{I}$. By this procedure one obtains interior systems for the quantities

$$
\left.\boldsymbol{v}^{(p)} \equiv \partial_{\rho}^{(p)} \boldsymbol{v}\right|_{\mathcal{I}},\left.\quad \phi^{(p)} \equiv \partial_{\rho}^{(p)} \phi\right|_{\mathcal{I}}
$$

The transport equations take the following form for $p \geq 1$ :

$$
\begin{aligned}
& \partial_{\tau} \boldsymbol{v}^{(p)}=\mathbf{K} \cdot \boldsymbol{v}^{(p)}+\sum_{j=0}^{p}\left(\begin{array}{l}
p \\
j
\end{array}\right)\left(\mathbf{Q}\left(\boldsymbol{v}^{(j)}, \boldsymbol{v}^{(p-j)}\right)+\mathbf{L}^{(j)} \cdot \boldsymbol{\phi}^{(p-j)}\right), \\
& \mathbf{E} \cdot \partial_{\tau} \boldsymbol{\phi}^{(p)}+\left(\mathbf{A}^{\mu}\right)^{(0)} \cdot \partial_{\mu} \boldsymbol{\phi}^{(p)}=\mathbf{F}\left(\boldsymbol{\Gamma}^{(0)}\right) \cdot \boldsymbol{\phi}^{(p)} \\
& +\sum_{j=1}^{p}\left(\begin{array}{l}
p \\
j
\end{array}\right)\left(\mathbf{F}\left(\boldsymbol{\Gamma}^{(j)}\right) \cdot \boldsymbol{\phi}^{(p-j)}-\left(\mathbf{A}^{\mu}\right)^{(j)} \cdot \partial_{\mu} \boldsymbol{\phi}^{(p-j)}\right) \\
& \left(\mathbf{B}^{\mu}\right)^{(0)} \cdot \partial_{\mu} \boldsymbol{\phi}^{(p)}=\mathbf{G}\left(\boldsymbol{\Gamma}^{(0)}\right) \cdot \phi^{(p)}+\sum_{j=1}^{p}\left(\begin{array}{l}
p \\
j
\end{array}\right)\left(\mathbf{G}\left(\boldsymbol{\Gamma}^{(j)}\right) \cdot \boldsymbol{\phi}^{(p-j)}-\left(\mathbf{B}^{\mu}\right)^{(j)} \cdot \partial_{\mu} \boldsymbol{\phi}^{(p-j)}\right) .
\end{aligned}
$$

Note that the non-homogeneous terms in the equations (18a)-(18c) depend on $\boldsymbol{v}^{\left(p^{\prime}\right)}, \phi^{\left(p^{\prime}\right)}$ for $0 \leq p^{\prime}<p$. Thus, if their values are known, then equations (18a)-(18b) constitutes an interior system of linear equations for $\boldsymbol{v}^{(p)}$ and $\phi^{(p)}$. The principal part of these equations is universal, in the sense that it is independent of the value of $p$. If the initial data on $\mathcal{C}_{a, \kappa}$ for the system (14)-15a is analytic - as it is the case in the present analysis - then suitable initial data for the interior system (18a)-18b can be obtained by repeated $\rho$-differentiation and evaluation on $\mathcal{I}^{0}$.

The language of jets is natural in the present context. For $p=0,1,2, \ldots$ and any sufficiently smooth (possibly vector valued) function $f$ defined on $\mathcal{M}_{a, \kappa}$, the sets of functions $\left\{f^{(0)}, f^{(1)}, \ldots\right.$, $\left.f^{(p)}\right\}$ on $\mathcal{I}$ will be denoted by $J_{\mathcal{I}}^{(p)}[f]$ and referred to as the jet order $p$ of $f$ on $\mathcal{I}$-and similarly with $\mathcal{I}$ replaced by $\mathcal{I}^{0}$. If $\mathbf{u}=(\boldsymbol{v}, \boldsymbol{\phi})$ is a solution to the equations (18a), (18b) and (18c), we refer to $J_{\mathcal{I}}^{(p)}[\mathbf{u}]$ as to the s-jet of $\mathbf{u}$ of order $p$ and to the data $J_{\mathcal{I}^{0}}^{(p)}[\mathbf{u}]$ as to the d-jet of $\mathbf{u}$ of order $p$. An s-jet $J_{\mathcal{I}}^{(p)}[\mathbf{u}]$ of order $p$ will be called regular on $\overline{\mathcal{I}} \equiv \mathcal{I} \cup \mathcal{I}^{+} \cup \mathcal{I}^{-}$if the corresponding functions extend smoothly to the critical sets $\mathcal{I}^{ \pm}$.

We note the following result of [22] which will be used in the sequel. 
Lemma 4. If the s-jets $J_{\mathcal{I}}^{(p-1)}[\boldsymbol{v}]$ and $J_{\mathcal{I}}^{(p-1)}[\phi]$ have polynomial dependence in $\tau$ for some $p \geq 1$, then $J_{\mathcal{I}}^{(p)}[\boldsymbol{v}]$ has also polynomial dependence in $\tau$.

\subsection{Decomposition in spherical harmonics}

Our analysis requires decomposing the entries of the vector unknowns $\boldsymbol{v}^{(p)}$ and $\boldsymbol{\phi}^{(p)}$ in terms of the functions $T_{i}{ }_{k}$. Following the discussion in [22, given a vector $\mathbf{u}^{(p)}=\left(u_{1}^{(p)}, \ldots, u_{N}^{(p)}\right)$ with entries in $\mathbb{R} \times \mathbb{S}^{2}$ and non-negative integers $q$ and $k=0, \ldots, 2 q$ one defines the sector $\mathfrak{S}_{q, k}\left[\mathbf{u}^{(p)}\right]$ as the collection of coefficients

$$
u_{i, p ; 2 q, k} \equiv(2 q+1) \int_{S U(2)} \bar{u}_{i}^{(p)} T_{2 q q-s}{ }^{k} \mathrm{~d} \mu,
$$

where $s$ is the spin-weight of $u_{i}^{(p)}, \mathrm{d} \mu$ is the Haar measure of $S U(2)$ and $\bar{u}_{i}^{(p)}$ denotes the complex conjugate of $u_{i}^{(p)}$. Furthermore, one defines

$$
\mathfrak{S}_{q}\left[\mathbf{u}^{(p)}\right] \equiv \bigcup_{k=0}^{2 q} \mathfrak{S}_{q, k}\left[\mathbf{u}^{(p)}\right]
$$

With this notation, a sector will be said to vanish if $\mathfrak{S}_{q}\left[\mathbf{u}^{(p)}\right]$. For convenience in the discussion, we introduce the following terminology:

- A coefficient $u_{i, p ; 2 q, k}$ will be said static if it depends only on the mass $m$ and on the terms in the expansions of $\stackrel{\circ}{U}$ and $\stackrel{\circ}{W}$. We will write $u_{i, p ; 2 q, k}=\stackrel{\circ}{u}_{i, p ; 2 q, k}$.

- The coefficient $u_{i, p ; 2 q, k}$ will be said to be Schwarzschildean if it only depends on $m$. In our gauge, Schwarzschildean terms can only arise in the sectors $\mathcal{S}_{0}$.

- A coefficient $u_{i, p ; 2 q, k}$ will be said to be non-static if it depends on the terms in the expansion of $\breve{W}$. We will write $u_{i, p ; 2 q, k}=\breve{u}_{i, p ; 2 q, k}$.

- A non-static coefficient $\breve{u}_{i, p ; 2 q, k}$ will be said to be a deviation from Schwarzschild if it only depends on $m$ and on the coefficients in the leading term of $\breve{W}$-i.e. $\breve{w}_{p_{\bullet}+1,2(p \bullet+1), k}$, $k=0, \ldots, 2(p \bullet+1)$.

The structure of the transport equations for the elements of the various sectors $\mathfrak{S}_{q, k}\left[\boldsymbol{v}^{(p)}\right]$ and $\mathfrak{S}_{q, k}\left[\phi^{(p)}\right]$ has been discussed in detail elsewhere - see [8, 11, 22. In particular, the degeneracy at the critical sets $\mathcal{I}^{ \pm}$implied by expression (17) is only relevant for sectors with $p \geq 2$. Thus, in the sequel it will always be assumed that $p \geq 2$. If we denote by ' differentiation with respect to $\tau$, the relevant transport equations are of the form:

$$
\begin{aligned}
& \boldsymbol{v}_{p ; 2 q, k}^{\prime}=\mathbf{K} \cdot \boldsymbol{v}_{p ; 2 q, k}+2 \mathbf{Q}\left(\boldsymbol{v}_{0 ; 0,0}, \boldsymbol{v}_{p ; 2 q, k}\right)+\mathbf{h}_{p ; 2 q, k}, \\
& (\mathbf{E}+\mathbf{A}) \cdot \boldsymbol{\phi}_{p ; 2 q, k}^{\prime}+\mathbf{A}_{p ; 2 q, k} \cdot \boldsymbol{\phi}_{p ; 2 q, k}=\mathbf{f}_{p ; 2 q, k}, \\
& \mathbf{B} \cdot \boldsymbol{\phi}_{p ; 2 q, k}^{\prime}+\mathbf{B}_{p ; 2 q, k} \cdot \boldsymbol{\phi}_{p ; 2 q, k}=\mathbf{g}_{p ; 2 q, k},
\end{aligned}
$$


where

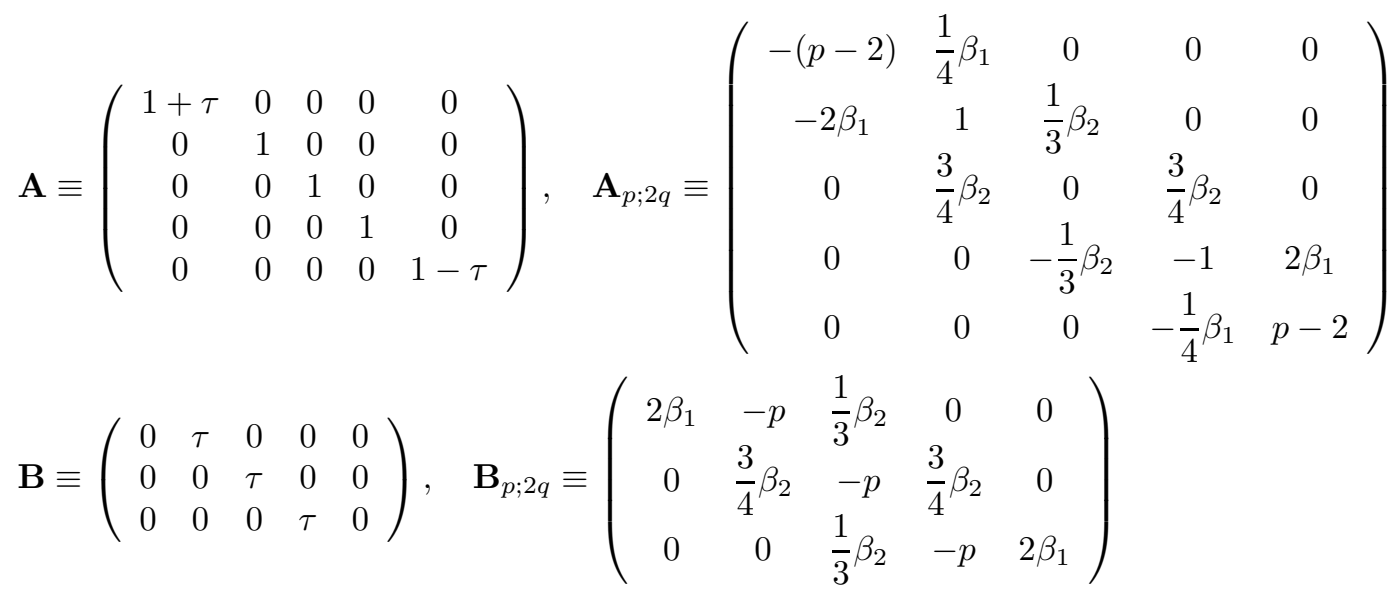

with

$$
\beta_{1} \equiv \sqrt{(q-1)(q+2)}, \quad \beta_{2} \equiv \sqrt{q(q+1)} .
$$

The terms $\mathbf{f}_{p ; 2 q, k}, \mathbf{g}_{p ; 2 q, k}, \mathbf{h}_{p ; 2 q, k}$ are calculated from the left hand sides of equations (18a)-(18c) using formula (9). Detailed expressions for certain values of the multiindex $(p ; q, k)$ will be given in the sequel.

\section{The cylinder at spatial infinity for static spacetimes}

As mentioned in the introduction, static initial data sets are expected to play a privileged role among the class of time symmetric initial data sets with a development which is asymptotically simple. This point of view is supported by the following result proved in [11] showing that the construction of the cylinder at spatial infinity described in the previous section is for static spacetimes as smooth as it is to be expected.

Theorem 2. For static vacuum solutions which are asymptotically flat, the construction of the cylinder at spatial infinity is analytic in the sense that there exists an a $>0$ and a frame for which all the fields, including the rescaled conformal Weyl tensor extend to analytic fields on some neighbourhood $\mathcal{N}$ of $\mathcal{I}$ in $\mathcal{M}_{\kappa, a}$. This statement depends neither on the particular choice of the conformal gauge used to prescribe the (analytic) free datum on $\mathcal{S}$ nor on the choice of $\kappa$.

Essential for our analysis is the following corollary:

Corollary 1. The solutions to the transport equations (18a)-(18c) for time symmetric static data given in the cn-gauge extend analytically through $\mathcal{I}^{ \pm}$for all orders $p$. Moreover, the solutions to the transport equations are polynomial in $\tau$.

Proof. Once analyticity has been asserted, the polynomial dependence of the solutions with respect to $\tau$ follows from an analysis of the possible solutions to the reduced equations - $\mathrm{cfr}$. the discussion in section 6.2 and 6.3 of $[22$.

\section{Properties of data sets which are static up to a given order}

In what follows we will discuss some properties of initial data sets which are static up to a certain order - in the sense of definition 2 These properties have mainly to do with the multipole structure of quantities associated to these initial data sets.

Given a quantity $u$, we will systematically write it as

$$
u=\stackrel{\imath}{u}+\breve{u},
$$


where, in the terminology of the previous section, $\stackrel{\leftrightarrow}{u}$ and $\breve{u}$ denote, respectively, its static and non-static parts. For quantities on the initial hypersurface $\mathcal{C}_{a, \kappa}$ we similarly write $u_{0}=\stackrel{\varkappa}{0}_{0}+\breve{u}_{0}$.

Let as before, $\mathbf{c}$ and $\boldsymbol{\Gamma}$ denote, respectively, the parts of the vectorial unknown $\boldsymbol{v}$ containing the independent components of the frame and connection coefficients. As mentioned in section 3.6. the expressions for the value of the frame and connection coefficients on the initial hypersurface $\mathcal{C}_{a, \kappa}$ are independent of $\Omega$. Thus, one has that:

Lemma 5. For data which is static up to order $p_{\bullet} \geq 0$ one has that

$$
\mathbf{c}_{0}^{(p)}=\stackrel{\circ}{\stackrel{\mathbf{c}}{0}_{0}^{(p)}}, \quad \boldsymbol{\Gamma}_{0}^{(p)}=\stackrel{\circ}{\boldsymbol{\Gamma}}_{0}^{(p)}
$$

for all $0 \leq p \leq p_{\bullet}$.

Crucial for our analysis is the behaviour of the data for the curvature spinors $\Theta_{A B C D}$ and $\phi_{A B C D}$. It is observed that

$$
\begin{aligned}
& \Omega=\rho^{2}-m \rho^{3}+\frac{3}{4} m^{2}+\mathcal{O}\left(\rho^{5}\right), \\
& D_{(A B} D_{C D)} \Omega=-6 m \epsilon_{A B C D}^{2}+12 m^{2} \epsilon_{A B C D}^{2} \rho^{2}+\mathcal{O}\left(\rho^{3}\right),
\end{aligned}
$$

from where it follows that the leading terms in these expressions are Schwarzschildean. The first non-Schwarzschildean contributions arise at orders $\mathcal{O}\left(\rho^{5}\right)$ and $\mathcal{O}\left(\rho^{3}\right)$, respectively - see e.g. 13. Using the expansions (20a)-(20b) together with formulae (16a)-(16b) one obtains after a long but straightforward computation the following:

Lemma 6. For data which is static up to order $p \bullet \geq 2$ one has that the curvature fields on $\mathcal{C}_{a, \kappa}$ satisfy

$$
\begin{aligned}
& \breve{\Theta}_{A B C D} \equiv \Theta_{A B C D}-\stackrel{\leftrightarrow}{\Theta}_{A B C D}=\mathcal{O}\left(\rho^{p}+2\right) \\
& \breve{\phi}_{A B C D} \equiv \phi_{A B C D}-\stackrel{\circ}{\phi}_{A B C D}=\mathcal{O}\left(\rho^{p \bullet+1}\right)
\end{aligned}
$$

Moreover, the terms

$$
\begin{array}{llll}
\breve{\Theta}_{A B C D}^{(p \cdot+1)}, & \breve{\Theta}_{A B C D}^{(p \cdot+2)}, & \breve{\Theta}_{A B C D}^{(p \cdot+3)}, & \breve{\Theta}_{A B C D}^{(p \cdot+4)}, \\
\breve{\phi}_{A B C D}^{(p \bullet+1)}, & \breve{\phi}_{A B C D}^{(p \cdot+2)}, & \breve{\phi}_{A B C D}^{(p \cdot+3)} &
\end{array}
$$

on $\mathcal{C}_{a, \kappa}$ are of the form of deviations from Schwarzschild data. On the other hand, $\breve{\phi}_{A B C D}^{\left(p_{\bullet}+4\right)}$ contains more general types of deviations.

An inspection of the explicit computations in [13] one obtains the following result.

Lemma 7. For a data which is static up to order $p_{\bullet} \geq 3$ one has that

$$
\begin{aligned}
& \boldsymbol{v}^{(0)}=\dot{\boldsymbol{v}}^{(0)}, \quad \boldsymbol{v}^{(1)}=\dot{\boldsymbol{v}}^{(1)}, \quad \boldsymbol{v}^{(2)}=\dot{\boldsymbol{v}}^{(2)}, \\
& \mathbf{L}^{(1)}=\stackrel{\circ}{\mathbf{L}}^{(1)}, \quad \mathbf{L}^{(2)}=\stackrel{\circ}{\mathbf{L}}^{(2)}, \quad \mathbf{L}^{(3)}=\stackrel{\circ}{\mathbf{L}}^{(3)}, \\
& \phi^{(0)}=\check{\phi}^{(0)}, \quad \phi^{(1)}=\dot{\phi}^{(1)}
\end{aligned}
$$

have only contributions to the sector $\mathfrak{S}_{0}$. On the other hand, the coefficients of

$$
\boldsymbol{v}^{(3)}=\dot{\boldsymbol{v}}^{(3)}, \quad \phi^{(2)}=\dot{\phi}^{(2)},
$$

contain contributions to the sectors $\mathfrak{S}_{0}$ and $\mathfrak{S}_{2}$.

Remark. In the previous lemma $\mathbf{L}$ denotes the linear matrix-valued function appearing in equation (14). It depends on the conformal factor $\Theta$ as given by (11) and on the 1-form $d_{A B}$ as given by (12).

A lenghty but straightforward computation using formulae (11) and (12) renders the following lemma.

Lemma 8. For data which is static up to order $p_{\bullet} \geq 3$ one has that

$$
\breve{\mathbf{L}}^{\left(\bullet_{\bullet}+1\right)}=0, \quad \breve{\mathbf{L}}^{\left(p_{\bullet}+2\right)}=0 .
$$




\section{Solutions to the transport equations for data which is static up to a certain order}

In this section we consider a time symmetric initial data set which is static up to order $p=p_{\bullet}+1$. The data can be parametrised in the form

$$
\boldsymbol{v}_{0}=\dot{\boldsymbol{v}}_{0}+\breve{\boldsymbol{v}}_{0}, \quad \phi_{0}=\stackrel{\circ}{\phi}_{0}+\breve{\boldsymbol{\phi}}_{0}
$$

where $\stackrel{\boldsymbol{v}}{0}_{0}, \dot{\phi}_{0}$ corresponds to exactly static initial data, while $\breve{\boldsymbol{v}}_{0}=0, \breve{\phi}_{0}=0$ if $W=m / 2$. A similar splitting can be applied to the quantities $\boldsymbol{v}^{(p)}, \boldsymbol{\phi}^{(p)}$. More precisely,

$$
\boldsymbol{v}^{(p)}=\dot{\boldsymbol{v}}^{(p)}+\breve{\boldsymbol{v}}^{(p)}, \quad \phi^{(p)}=\stackrel{\circ}{\boldsymbol{\phi}}(p)^{(p)} \breve{\boldsymbol{\phi}}^{(p)}, \quad p \geq 0 .
$$

One has the following result.

Lemma 9. For initial data which is static up to order $p=p_{\bullet}$, one has that

$$
\breve{\boldsymbol{v}}^{(p)}=0, \quad 0 \leq p \leq p \bullet+1,
$$

and

$$
\breve{\phi}^{(p)}=0, \quad 0 \leq p \leq p_{\bullet} .
$$

In other words, the first deviations from static evolution appear in $\phi^{\left(p_{\bullet}+1\right)}$.

Proof. One first notes that

$$
\breve{\boldsymbol{v}}_{0}^{(p)}=0, \quad p \leq p_{\bullet}+1
$$

and

$$
\breve{\phi}_{0}^{(p)}=0, \quad p \leq p_{\bullet} .
$$

One then argues by induction. It is clear that

$$
\breve{\boldsymbol{v}}^{(0)}=0, \quad \breve{\phi}^{(0)}=0 .
$$

Now, given $p$ such that $1 \leq p \leq p_{\bullet}$, assume that $\breve{\boldsymbol{v}}^{\left(p^{\prime}\right)}=0$ and $\breve{\boldsymbol{\phi}}^{\left(p^{\prime}\right)}=0$ for $0 \leq p^{\prime}<p$. Substitution of the Ansätze $\boldsymbol{v}^{(j)}=\dot{\boldsymbol{v}}^{(j)}+\breve{\boldsymbol{v}}^{(j)}, \phi^{(j)}=\dot{\phi}^{(j)}+\breve{\phi}^{(j)}, j=0, \ldots, p$ into the $v$ transport equation (18a) renders the equation

$$
\begin{aligned}
& \partial_{\tau} \dot{\boldsymbol{v}}^{(p)}+\partial_{\tau} \breve{\boldsymbol{v}}^{(p)}=\mathbf{K} \cdot \dot{\boldsymbol{v}}^{(p)}+\mathbf{K} \cdot \breve{\boldsymbol{v}}^{(p)}
\end{aligned}
$$

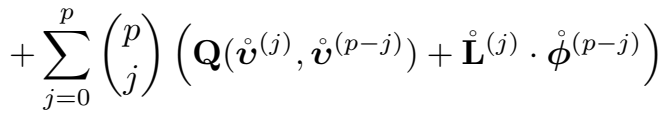

$$
\begin{aligned}
& +\sum_{j=0}^{p}\left(\begin{array}{l}
p \\
j
\end{array}\right)\left(\mathbf{Q}\left(\dot{\boldsymbol{v}}^{(j)}, \breve{\boldsymbol{v}}^{(p-j)}\right)+\stackrel{\circ}{\mathbf{L}}^{(j)} \cdot \breve{\boldsymbol{\phi}}^{(p-j)}\right)
\end{aligned}
$$

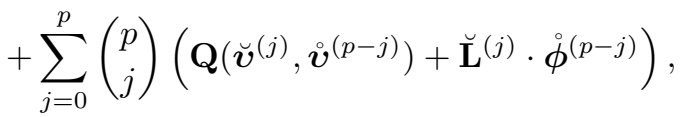

$$
\begin{aligned}
& +\sum_{j=0}^{p}\left(\begin{array}{l}
p \\
j
\end{array}\right)\left(\mathbf{Q}\left(\breve{\boldsymbol{v}}^{(j)}, \breve{\boldsymbol{v}}^{(p-j)}\right)+\breve{\mathbf{L}}^{(j)} \cdot \breve{\boldsymbol{\phi}}^{(p-j)}\right) .
\end{aligned}
$$

Using the induction hypothesis and

$$
\partial_{\tau} \stackrel{\boldsymbol{v}}{(p)}^{(p)} \mathbf{K} \cdot \stackrel{\boldsymbol{v}}{(p)}^{p}+\sum_{j=0}^{p}\left(\begin{array}{c}
p \\
j
\end{array}\right)\left(\mathbf{Q}\left(\stackrel{\boldsymbol{v}}{(j)}^{\left(\boldsymbol{v}^{(p-j)}\right.}\right)+\stackrel{\circ}{\mathbf{L}}^{(j)} \cdot \stackrel{\circ}{\phi}^{(p-j)}\right),
$$

one finds that

$$
\partial_{\tau} \breve{\boldsymbol{v}}^{(p)}=\mathbf{K} \cdot \breve{\boldsymbol{v}}^{(p)}+\mathbf{Q}\left(\dot{\boldsymbol{v}}^{(0)}, \breve{\boldsymbol{v}}^{(p)}\right)+\mathbf{Q}\left(\breve{\boldsymbol{v}}^{(p)}, \dot{\boldsymbol{v}}^{(0)}\right)-\stackrel{\circ}{\mathbf{L}}^{(0)} \cdot \breve{\boldsymbol{\phi}}^{(p)}-\breve{\mathbf{L}}^{(p)} \cdot \stackrel{\circ}{\phi}^{(0)}
$$


As this last equation is homogeneous in the unknown $\breve{\boldsymbol{v}}^{(p)}$, the initial condition $\breve{\boldsymbol{v}}_{0}^{(p)}=0$ implies $\breve{\boldsymbol{v}}^{(p)}=0$. A similar argument leads to the following equation for $\breve{\boldsymbol{\phi}}^{(p)}$ :

$$
\sqrt{2} \mathbf{E} \cdot \partial_{\tau} \breve{\boldsymbol{\phi}}^{(p)}+\left(\AA^{\mu}\right)^{(0)} \cdot \partial_{\mu} \breve{\boldsymbol{\phi}}^{(p)}=\mathbf{F}^{(0)} \cdot \breve{\boldsymbol{\phi}}^{(p)} .
$$

Again, the homogeneity of this last equation, together with the initial condition $\breve{\phi}_{0}^{(p)}=0$ renders $\breve{\phi}^{(p)}=0$. Thus, one has that $\breve{\boldsymbol{v}}^{(p)}=0, \breve{\boldsymbol{\phi}}^{(p)}=0,0 \leq p \leq p$. The argument can be repeated for $\breve{\boldsymbol{v}}^{(p \bullet+1)}$ as $\breve{\boldsymbol{v}}_{0}^{\left(p_{\bullet}+1\right)}=0$.

The ideas of the proof of Lemma 9 will be used to study with some detail the solutions to the sector $\mathfrak{S}_{p_{\bullet}+1}$ transport equations for the orders $p_{\bullet}+1, p_{\bullet}+2, p_{\bullet}+3$ and $p_{\bullet}+4$.

\subsection{The transport equations at order $p=p_{\bullet}+1$}

From Lemma 9 one knows that $\boldsymbol{v}^{\left(\boldsymbol{\bullet}_{\bullet}+1\right)}=\dot{\boldsymbol{v}}^{\left(\boldsymbol{\bullet}_{\bullet}+1\right)}$. Thus, at this order one only needs to study the solutions to the Bianchi transport equations.

Substitution of the Ansätze

$$
\boldsymbol{v}^{(j)}=\dot{\boldsymbol{v}}^{(j)}+\breve{\boldsymbol{v}}^{(j)}, \quad \phi^{(j)}=\stackrel{\circ}{\boldsymbol{\phi}}^{(j)}+\breve{\boldsymbol{\phi}}^{(j)}, \quad 0 \leq j \leq p \bullet+1
$$

into equations (18b)-(18c) with $p=p_{\bullet}+1$ and using Lemma 9 one obtains the following equations for $\breve{\phi}^{(p \bullet+1)}$ :

$$
\begin{aligned}
& \sqrt{2} \mathbf{E} \cdot \partial_{\tau} \breve{\boldsymbol{\phi}}^{(p)}+\left(\stackrel{\AA}{\mathbf{A}}^{\mu}\right)^{(0)} \cdot \partial_{\mu} \breve{\boldsymbol{\phi}}^{(p)}=\stackrel{\circ}{\mathbf{F}}^{(0)} \cdot \breve{\boldsymbol{\phi}}^{(p)} \\
& \left(\stackrel{\mathbf{B}}{ }^{\mu}\right)^{(0)} \cdot \partial_{\mu} \breve{\boldsymbol{\phi}}^{(p)}=\stackrel{\mathbf{G}}{ }^{(0)} \cdot \breve{\boldsymbol{\phi}}^{(p)} .
\end{aligned}
$$

We now focus on the sector $\mathfrak{S}_{p \bullet+1}$ of these equations. Recalling that $\left(\AA^{\mu}\right)^{(0)},\left(\stackrel{\mathbf{B}}{\mu}^{\mu}\right)^{(0)}, \stackrel{\circ}{\mathbf{F}}^{(0)}, \stackrel{\circ}{\mathbf{G}}^{(0)}$ only contain the sector $\mathfrak{S}_{0}$, one obtains the matricial equations

$$
\begin{aligned}
& \mathbf{A} \cdot \breve{\boldsymbol{\phi}}_{p_{\bullet}+1 ; 2\left(p_{\bullet}+1\right)}^{\prime}+\mathbf{A}_{p_{\bullet}+1 ; 2\left(p_{\bullet}+1\right)} \cdot \breve{\boldsymbol{\phi}}_{p_{\bullet}+1 ; 2\left(p_{\bullet}+1\right)}=0, \\
& \mathbf{B} \cdot \breve{\boldsymbol{\phi}}_{p_{\bullet}+1 ; 2\left(p_{\bullet}+1\right)}^{\prime}+\mathbf{B}_{p_{\bullet}+1 ; 2\left(p_{\bullet}+1\right)} \cdot \breve{\boldsymbol{\phi}}_{p_{\bullet}+1 ; 2\left(p_{\bullet}+1\right)}=0,
\end{aligned}
$$

where $\mathbf{A}, \mathbf{A}_{p_{\bullet}+1 ; 2\left(p_{\bullet}+1\right)}, \mathbf{B}$ and $\mathbf{B}_{p_{\bullet}+1 ; 2\left(p_{\bullet}+1\right)}$ are the matrices given by (19a)-(19b). For the sake of the simplicity of the presentation, the subindex $k$ has been omitted from these and most of the subsequent equations. A lengthy but straightforward computation shows that the initial data for these equations is given by

$$
\breve{\phi}_{p_{\bullet}+1 ; 2(p \bullet+1), k}(0)=\left(\breve{a}_{0, k}, \breve{a}_{1, k}, \breve{a}_{2, k}, \breve{a}_{1, k}, \breve{a}_{0, k}\right),
$$

with $k=0, \ldots, 2(p \bullet+1)$ and

$$
\begin{aligned}
\breve{a}_{0, k} & \equiv-\sqrt{p_{\bullet}\left(p_{\bullet}+1\right)\left(p_{\bullet}+2\right)\left(p_{\bullet}+3\right)} \breve{w}_{p_{\bullet}+1 ; 2\left(p_{\bullet}+1\right), k}, \\
\breve{a}_{1, k} & \equiv-4\left(p_{\bullet}+3\right) \sqrt{\left(p_{\bullet}+1\right)\left(p_{\bullet}+2\right)} \breve{w}_{p_{\bullet}+1 ; 2\left(p_{\bullet}+1\right), k}, \\
\breve{a}_{2, k} & \equiv-6\left(p_{\bullet}+2\right)\left(p_{\bullet}+3\right) \breve{w}_{p_{\bullet}+1 ; 2\left(p_{\bullet}+1\right), k}
\end{aligned}
$$

The previous equations lead to the following crucial observation:

Observation 1. Equations (21a)-(21b) and their corresponding initial data are formally identical to the sector $\mathfrak{S}_{p_{\bullet}+1}\left[\phi^{\left(p_{\bullet}+1\right)}\right]$ transport equations for data which is Schwarzschildean up to order $p_{\bullet}+1$; the solutions are, therefore, also formally identical to those obtained in [22].

As a consequence of the latter observation one obtains from the analysis in [22] that:

$$
\breve{\phi}_{p_{\bullet}+1 ; 2\left(p_{\bullet}+1\right)}=\tilde{\boldsymbol{\varphi}}_{p_{\bullet}+1}(\tau)(1-\tau)^{p_{\bullet}-1}(1+\tau)^{p_{\bullet}-1},
$$

with $\tilde{\varphi}_{p_{\bullet}+1}(\tau)$ having entries which are polynomials of degree 4 in $\tau$. In particular,

$$
\tilde{\boldsymbol{\varphi}}_{p_{\bullet}+1}(0)=\breve{\boldsymbol{\phi}}_{p_{\bullet}+1 ; 2(p \bullet+1)}(0)
$$

as given by (22).

Combining this analysis with Corollary 1 one finds the following result: 
Proposition 3. The solution to the transport equations at spatial infinity at order $p_{\bullet}+1$ for data which is static up to order $p_{\bullet}$ are polynomial in $\tau$. Hence, they extend analytically through $\tau= \pm 1$.

\subsection{The transport equations at order $p=p_{\bullet}+2$}

Using Lemma 4 one finds that the solutions $\boldsymbol{v}^{\left(p_{\bullet}+2\right)}$ to the order $p_{\bullet}+2 \boldsymbol{v}$-transport equations are polynomial in $\tau$ given that $\boldsymbol{v}^{(p)}$ and $\phi^{(p)}$ for $0 \leq p \leq p_{\bullet}+1$ are polynomial in $\tau$. However, we require more precise information.

Again, we consider the transport equations (18a) for the order $p_{\bullet}+2$. The substitution of the Ansätze

$$
\boldsymbol{v}^{(p)}=\dot{\boldsymbol{v}}^{(p)}+\breve{\boldsymbol{v}}^{(p)}, \quad \phi^{(p)}=\stackrel{\circ}{\boldsymbol{\phi}}^{(p)}+\breve{\boldsymbol{\phi}}^{(p)}, \quad 0 \leq p \leq p_{\bullet}+2,
$$

and considerations similar to the ones used for the order $p_{\bullet}+1$ lead to the following equations for $\breve{\boldsymbol{v}}^{(p \bullet+2)}$ and $\breve{\boldsymbol{\phi}}^{(p \bullet+2)}$ :

$$
\begin{aligned}
& \partial_{\tau} \breve{\boldsymbol{v}}^{(p \bullet+2)}=\mathbf{K} \cdot \breve{\boldsymbol{v}}^{\left(p_{\bullet}+2\right)}+\mathbf{Q}\left(\dot{\boldsymbol{v}}^{(0)}, \breve{\boldsymbol{v}}^{\left(p_{\bullet}+2\right)}\right)+\mathbf{Q}\left(\breve{\boldsymbol{v}}^{\left(p_{\bullet}+2\right)}, \dot{\boldsymbol{v}}^{(0)}\right)+\stackrel{\circ}{\mathbf{L}}^{(1)} \cdot \breve{\boldsymbol{\phi}}^{(p \bullet+1)}, \\
& \sqrt{2} \mathbf{E} \cdot \partial_{\tau} \breve{\boldsymbol{\phi}}^{\left(p_{\bullet}+2\right)}+\left(\stackrel{\circ}{ }^{\mu}\right)^{(0)} \cdot \partial_{\mu} \breve{\boldsymbol{\phi}}^{\left(p_{\bullet}+2\right)}=\stackrel{\circ}{\mathbf{F}}^{(0)} \cdot \breve{\boldsymbol{\phi}}_{p_{\bullet}+2}+\left(p_{\bullet}+2\right) \stackrel{\circ}{\mathbf{F}}^{(1)} \cdot \breve{\boldsymbol{\phi}}^{\left(p_{\bullet}+1\right)} \\
& +\breve{\mathbf{F}}^{\left(p_{\bullet}+2\right)} \cdot \stackrel{\boldsymbol{\phi}}{(0)}^{(0)}\left(p_{\bullet}+2\right)\left(\stackrel{\circ}{ }^{\mu}\right)^{(1)} \cdot \partial_{\mu} \breve{\boldsymbol{\phi}}^{\left(p_{\bullet}+1\right)}, \\
& \left(\stackrel{\circ}{\mathbf{B}}^{\mu}\right)^{(0)} \partial_{\mu} \breve{\boldsymbol{\phi}}^{\left(p_{\bullet}+2\right)}=\stackrel{\circ}{\mathbf{G}}^{(0)} \cdot \breve{\boldsymbol{\phi}}_{p_{\bullet}+2}+\left(p_{\bullet}+2\right) \stackrel{\circ}{\mathbf{G}}^{(1)} \cdot \breve{\boldsymbol{\phi}}^{\left(p_{\bullet}+1\right)} \\
& +\breve{\mathbf{G}}^{\left(p_{\bullet}+2\right)} \cdot \stackrel{\circ}{\boldsymbol{\phi}}^{(0)}-\left(p_{\bullet}+2\right)\left(\stackrel{\circ}{\mathbf{B}}^{\mu}\right)^{(1)} \cdot \partial_{\mu} \breve{\boldsymbol{\phi}}^{(p \bullet+1)} \text {. }
\end{aligned}
$$

Again, as a consequence of Lemma 7 one has that

$$
\stackrel{\circ}{\boldsymbol{v}}^{(0)}, \quad \stackrel{\circ}{\phi}^{(0)}, \quad \stackrel{\circ}{\mathbf{L}}^{(1)}
$$

contain only contributions to the sector $\mathfrak{S}_{0}$ so that one obtains directly the following equations for the components of $\mathfrak{S}_{p_{\bullet}+1}\left[\breve{\boldsymbol{v}}^{(p \bullet+2)}, \breve{\boldsymbol{\phi}}^{(p \bullet+2)}\right]$ :

$$
\begin{aligned}
& \breve{\boldsymbol{v}}_{p_{\bullet}+2 ; 2\left(p_{\bullet}+1\right)}^{\prime}=\mathbf{K} \breve{\boldsymbol{v}}_{p_{\bullet}+2 ; 2\left(p_{\bullet}+1\right)}+\mathbf{Q}\left(\stackrel{\circ}{0 ; 0}_{0}, \breve{\boldsymbol{v}}_{p_{\bullet}+2 ; 2\left(p_{\bullet}+1\right)}\right) \\
& +\mathbf{Q}\left(\breve{\boldsymbol{v}}_{p \bullet+2 ; 2(p \bullet+1)}, \stackrel{\circ}{0 ; 0}\right)+\stackrel{\circ}{\mathbf{L}}_{1 ; 0} \cdot \breve{\boldsymbol{\phi}}_{p \bullet+1 ; 2(p \bullet+1)}, \\
& (\mathbf{E}+\mathbf{A}) \cdot \breve{\boldsymbol{\phi}}_{p_{\bullet}+2 ; 2\left(p_{\bullet}+1\right)}^{\prime}+\mathbf{A}_{p_{\bullet}+2 ; 2(p \bullet+1)} \cdot \breve{\boldsymbol{\phi}}_{p_{\bullet}+2 ; 2(p \bullet+1)}=\stackrel{\circ}{\mathbf{F}}_{0 ; 0} \cdot \breve{\boldsymbol{\phi}}_{p_{\bullet}+2 ; 2(p \bullet+1)} \\
& +\left(p_{\bullet}+2\right) \stackrel{\circ}{\mathbf{F}}_{1 ; 0} \cdot \breve{\boldsymbol{\phi}}_{p_{\bullet}+1 ; 2(p \bullet+1)}+\breve{\mathbf{F}}_{p_{\bullet}+2 ; 2(p \bullet+1)} \cdot{\stackrel{\circ}{\boldsymbol{\phi}_{0 ; 0}}}-\left(p_{\bullet}+2\right) \stackrel{\circ}{\mathbf{A}}_{1 ; 0} \cdot \breve{\boldsymbol{\phi}}_{p_{\bullet}+1 ; 2(p \bullet+1)},
\end{aligned}
$$

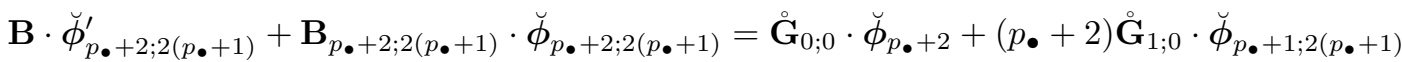

$$
\begin{aligned}
& +\breve{\mathbf{G}}_{p_{\bullet}+2 ; 2\left(p_{\bullet}+1\right)} \cdot{\stackrel{\circ}{\boldsymbol{\phi}_{0 ; 0}}}-\left(p_{\bullet}+2\right){\stackrel{\circ}{\mathbf{B}_{1 ; 0}}} \cdot \breve{\boldsymbol{\phi}}_{p_{\bullet}+1 ; 2(p \bullet+1)} \cdot
\end{aligned}
$$

Using Lemma 9, one sees that the components in

$$
\stackrel{\circ}{\boldsymbol{v}}_{0 ; 0}, \stackrel{\circ}{\mathbf{L}}_{1 ; 0}, \stackrel{\circ}{\mathbf{F}}_{0 ; 0}, \stackrel{\circ}{\mathbf{G}}_{0 ; 0},
$$

are exactly Minkowskian while the components in

$$
\stackrel{\circ}{\phi}_{0 ; 0}, \stackrel{\circ}{\mathbf{A}}_{1 ; 0}, \stackrel{\circ}{\mathbf{B}}_{1 ; 0}, \stackrel{\circ}{\mathbf{F}}_{1 ; 0}, \stackrel{\circ}{\mathbf{G}}_{1 ; 0} \text {, }
$$

are exactly Schwarzschildean - that is, they depend only on the mass $m$. On the other hand, as already seen, the components in $\breve{\phi}_{p_{\bullet}+1 ; 2\left(p_{\bullet}+1\right)}$ depend only on $\breve{w}_{p_{\bullet}+1 ; 2(p \bullet+1), k}$. This leads to the following crucial observation at this order:

Observation 2. The equations (23a) -(23c) are formally identical to the order $p_{\bullet}+2$ transport equations for initial data sets which are Schwarzschildean up to order $p_{\bullet}$; similarly, due to Lemma [6] the initial data set is formally also of the form of a perturbation of Schwarzschild.

As a consequence of the previous discussion one can directly use the analysis and results of 22 . to directly conclude that the solutions $\breve{\boldsymbol{v}}_{p_{\bullet}+2 ; 2(p \bullet+1)}$, and $\breve{\boldsymbol{\phi}}_{p \bullet+; 2(p \bullet+1)}$, to equations (23a)-(23c) are polynomial in $\tau$. Combining this observation with Corollary 1 one has that:

Proposition 4. The solutions to the order $p_{\bullet}+2$ transport equations (18a)-(18c) are polynomial in $\tau$ and, thus, extend analytically through $\tau= \pm 1$. 


\subsection{The transport equations at order $p=p_{\bullet}+3$}

We now adapt the procedure discussed in the previous section to the analysis of the order $p_{\bullet}+3$ transport equations. As in the previous order the polynomial dependence in $\tau$ follows directly from Lemma 4 once one knows that the entries in $\phi^{\left(p_{\bullet}+2\right)}$ are polynomial. Further detailed information will follow from the analysis of the sector $\mathfrak{S}_{p_{\bullet}+1}$.

The analysis for this order is similar to that for orders $p_{\bullet}+1$ and $p_{\bullet}+2$. Substitution of the Ansätze

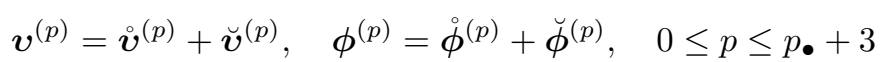

into the transport equations (18a)-(18c) leads to the following equations for $\breve{\boldsymbol{v}}^{\left(p_{\bullet}+3\right)}$ and $\breve{\phi}^{(p \bullet+3)}$ :

$$
\begin{aligned}
& \partial_{\tau} \breve{\boldsymbol{v}}^{\left(p_{\bullet}+3\right)}=\mathbf{K} \cdot \breve{\boldsymbol{v}}^{(p \bullet+3)}+\mathbf{Q}\left(\stackrel{\boldsymbol{v}}{(0)}^{(0)} \breve{\boldsymbol{v}}^{(p \bullet+3)}\right)+\mathbf{Q}\left(\breve{\boldsymbol{v}}^{\left(p_{\bullet}+3\right)}, \stackrel{\boldsymbol{v}}{(0)}\right)^{(0)}
\end{aligned}
$$

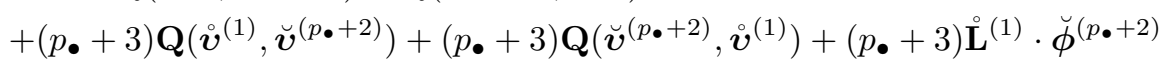

$$
\begin{aligned}
& +\frac{1}{2}\left(p_{\bullet}+3\right)\left(p_{\bullet}+2\right) \stackrel{\circ}{\mathbf{L}}^{(2)} \cdot \breve{\phi}^{\left(p_{\bullet}+1\right)}+\breve{\mathbf{L}}^{\left(p_{\bullet}+3\right)} \cdot \stackrel{\circ}{\phi}^{(0)} \text {, } \\
& \sqrt{2} \mathbf{E} \partial_{\tau} \breve{\boldsymbol{\phi}}^{\left(p_{\bullet}+3\right)}+\left(\stackrel{\AA}{ }^{\mu}\right)^{(0)} \cdot \partial_{\mu} \breve{\boldsymbol{\phi}}^{\left(p_{\bullet}+3\right)}=\stackrel{\circ}{\mathbf{F}}^{(0)} \cdot \breve{\boldsymbol{\phi}}_{p_{\bullet}+3}+\left(p_{\bullet}+3\right) \stackrel{\circ}{\mathbf{F}}^{(1)} \cdot \breve{\boldsymbol{\phi}}^{(p \bullet+2)} \\
& +\frac{1}{2}\left(p_{\bullet}+3\right)\left(p_{\bullet}+2\right) \stackrel{\circ}{\mathbf{F}}^{(2)} \cdot \breve{\boldsymbol{\phi}}^{\left(p_{\bullet}+1\right)}+\left(p_{\bullet}+3\right) \breve{\mathbf{F}}^{\left(p_{\bullet}+2\right)} \cdot \stackrel{\circ}{\boldsymbol{\phi}}^{(1)}+\breve{\mathbf{F}}^{\left(p_{\bullet}+3\right)} \cdot \stackrel{\circ}{\boldsymbol{\phi}}^{(0)} \\
& -\left(p_{\bullet}+3\right)\left(\AA^{\mu}\right)^{(1)} \cdot \partial_{\mu} \breve{\boldsymbol{\phi}}^{\left(p_{\bullet}+2\right)}-\frac{1}{2}\left(p_{\bullet}+3\right)\left(p_{\bullet}+2\right)\left(\AA^{\mu}\right)^{(2)} \cdot \partial_{\mu} \breve{\boldsymbol{\phi}}^{\left(p_{\bullet}+1\right)} \\
& -\left(p_{\bullet}+3\right)\left(\breve{\mathbf{A}}^{\mu}\right)^{(p \bullet+2)} \cdot \partial_{\mu} \stackrel{\circ}{\phi}^{(1)}-\left(\breve{\mathbf{A}}^{\mu}\right)^{(p \bullet+3)} \cdot \partial_{\mu} \stackrel{\circ}{\phi}^{(0)}, \\
& \left(\mathbf{B}^{\mu}\right)^{(0)} \partial_{\mu} \breve{\boldsymbol{\phi}}^{(p \bullet+3)}=\stackrel{\circ}{\mathbf{G}}^{(0)} \cdot \breve{\boldsymbol{\phi}}_{p_{\bullet}+3}+\left(p_{\bullet}+3\right) \stackrel{\circ}{\mathbf{G}}^{(1)} \cdot \breve{\boldsymbol{\phi}}^{\left(p_{\bullet}+2\right)}+\frac{1}{2}\left(p_{\bullet}+3\right)\left(p_{\bullet}+2\right) \stackrel{\circ}{\mathbf{G}}^{(2)} \cdot \breve{\boldsymbol{\phi}}^{(p \bullet+1)} \\
& +\breve{\mathbf{G}}^{\left(p_{\bullet}+3\right)} \cdot \stackrel{\circ}{\boldsymbol{\phi}}^{(0)}+\left(p_{\bullet}+3\right) \breve{\mathbf{G}}^{\left(p_{\bullet}+2\right)} \cdot \stackrel{\circ}{\boldsymbol{\phi}}^{(1)} \\
& -\left(p_{\bullet}+3\right)\left(\stackrel{\circ}{\mathbf{B}}^{\mu}\right)^{(1)} \cdot \partial_{\mu} \breve{\boldsymbol{\phi}}^{\left(p_{\bullet}+2\right)}-\frac{1}{2}\left(p_{\bullet}+3\right)\left(p_{\bullet}+2\right)\left(\stackrel{\circ}{ }^{\mu}\right)^{(2)} \cdot \partial_{\mu} \breve{\boldsymbol{\phi}}^{\left(p_{\bullet}+1\right)} \\
& -\left(p_{\bullet}+3\right)\left(\breve{\mathbf{B}}^{\mu}\right)^{\left(p_{\bullet}+2\right)} \cdot \partial_{\mu} \stackrel{\circ}{\phi}^{(1)}-\left(\breve{\mathbf{B}}^{\mu}\right)^{\left(p_{\bullet}+3\right)} \cdot \partial_{\mu} \stackrel{\circ}{\boldsymbol{\phi}}^{(0)},
\end{aligned}
$$

Using Lemma[7 one obtains the following equations for the components of $\mathfrak{S}_{p_{\bullet}+1}\left[\breve{\boldsymbol{v}}^{\left(p_{\bullet}+3\right)}, \breve{\boldsymbol{\phi}}^{\left(p_{\bullet}+3\right)}\right]$ :

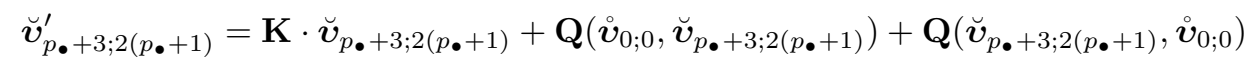

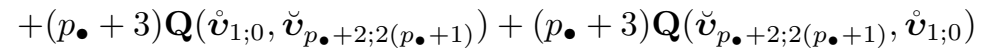

$$
\begin{aligned}
& +\left(p_{\bullet}+3\right) \stackrel{\circ}{\mathbf{L}}_{1 ; 0} \cdot \breve{\boldsymbol{\phi}}_{p \bullet+2 ; 2\left(p_{\bullet}+1\right)}+\frac{1}{2}\left(p_{\bullet}+3\right)\left(p_{\bullet}+2\right) \stackrel{\circ}{\mathbf{L}}_{2 ; 0} \cdot \breve{\boldsymbol{\phi}}_{p_{\bullet}+1 ; 2(p \bullet+1)} \\
& +\stackrel{\circ}{\mathbf{L}}_{p \bullet+3 ; 2\left(p_{\bullet}+1\right)} \cdot \stackrel{\circ}{\phi}_{0 ; 0},
\end{aligned}
$$

$$
\begin{aligned}
& (\mathbf{E}+\mathbf{A}) \cdot \breve{\boldsymbol{\phi}}_{p \bullet+3 ; 2(p \bullet+1)}^{\prime}+\mathbf{A}_{p_{\bullet}+3 ; 2(p \bullet+1)} \cdot \breve{\boldsymbol{\phi}}_{p_{\bullet}+3 ; 2\left(p_{\bullet}+1\right)}=\stackrel{\circ}{\mathbf{F}}_{0 ; 0} \cdot \breve{\boldsymbol{\phi}}_{p \bullet+3 ; 2(p \bullet+1)}+\left(p_{\bullet}+3\right){\stackrel{\circ}{\mathbf{F}_{1 ; 0}}} \cdot \breve{\boldsymbol{\phi}}_{p_{\bullet}+2 ; 2\left(p_{\bullet}+1\right)} \\
& +\frac{1}{2}\left(p_{\bullet}+3\right)\left(p_{\bullet}+2\right) \stackrel{\circ}{\mathbf{F}}_{2 ; 0} \cdot \breve{\boldsymbol{\phi}}_{p_{\bullet}+1 ; 2(p \bullet+1)}+\left(p_{\bullet}+3\right) \breve{\mathbf{F}}_{p_{\bullet}+2 ; 2\left(p_{\bullet}+1\right)} \cdot \stackrel{\circ}{\boldsymbol{\phi}}_{1 ; 0} \\
& +\breve{\mathbf{F}}_{p_{\bullet}+3 ; 2(p \bullet+1)} \cdot \stackrel{\circ}{0 ; 0} \\
& -\left(p_{\bullet}+3\right) \stackrel{\AA}{1 ; 0}_{1 ;} \cdot \breve{\boldsymbol{\phi}}_{p_{\bullet}+2 ; 2(p \bullet+1)}^{\prime}-\left(p_{\bullet}+3\right) \stackrel{\AA}{\mathbf{A}}_{1 ; 0}^{+} \cdot \breve{\boldsymbol{\phi}}_{p_{\bullet}+2 ; 2\left(p_{\bullet}+1\right)}-\left(p_{\bullet}+3\right) \AA_{1 ; 0}^{-} \cdot \breve{\boldsymbol{\phi}}_{p_{\bullet}+2 ; 2(p \bullet+1)} \\
& -\frac{1}{2}\left(p_{\bullet}+3\right)\left(p_{\bullet}+2\right) \AA_{2 ; 0} \cdot \breve{\boldsymbol{\phi}}_{p \bullet+1 ; 2(p \bullet+1)}^{\prime}-\frac{1}{2}\left(p_{\bullet}+3\right)\left(p_{\bullet}+2\right) \stackrel{\mathbf{A}}{2 ; 0}_{\bullet}^{+} \cdot \breve{\boldsymbol{\phi}}_{p \bullet+1 ; 2(p \bullet+1)} \\
& -\frac{1}{2}\left(p_{\bullet}+3\right)\left(p_{\bullet}+2\right) \stackrel{\AA}{\mathbf{A}}_{2 ; 0}^{-} \cdot \breve{\boldsymbol{\phi}}_{p_{\bullet}+1 ; 2(p \bullet+1)}-\left(p_{\bullet}+3\right) \breve{\mathbf{A}}_{p_{\bullet}+2 ; 2(p \bullet+1)} \cdot \stackrel{\circ}{1 ; 0}^{\prime} \text {, }
\end{aligned}
$$

$$
\begin{aligned}
& \mathbf{B} \cdot \breve{\boldsymbol{\phi}}_{p \bullet+3 ; 2\left(p_{\bullet}+1\right)}^{\prime}+\mathbf{B}_{p_{\bullet}+3 ; 2(p \bullet+1)} \cdot \breve{\boldsymbol{\phi}}_{p_{\bullet}+3 ; 2(p \bullet+1)}=\stackrel{\mathrm{G}}{0 ; 0}_{\bullet} \cdot \breve{\boldsymbol{\phi}}_{p_{\bullet}+3 ; 2(p \bullet+1)}+(p \bullet+3) \stackrel{\circ}{\mathbf{G}}_{1 ; 0} \cdot \breve{\boldsymbol{\phi}}_{p_{\bullet}+2 ; 2(p \bullet+1)} \\
& +\frac{1}{2}\left(p_{\bullet}+3\right)\left(p_{\bullet}+2\right) \stackrel{\mathbf{G}}{2 ; 0}_{\boldsymbol{\phi}_{p_{\bullet}+1 ; 2(p \bullet+1)}}+\breve{\mathbf{G}}_{p_{\bullet}+3 ; 2\left(p_{\bullet}+1\right)} \cdot \stackrel{\circ}{\boldsymbol{\phi}} 0 ; 0+\left(p_{\bullet}+3\right) \breve{\mathbf{G}}_{p_{\bullet}+2 ; 2(p \bullet+1)} \cdot{\stackrel{\circ}{\boldsymbol{\phi}_{1 ; 0}}} \\
& -\left(p_{\bullet}+3\right) \stackrel{\circ}{\mathbf{B}}_{1 ; 0} \cdot \breve{\phi}_{p_{\bullet}+2 ; 2(p \bullet+1)}^{\prime}-\left(p_{\bullet}+3\right) \stackrel{\circ}{\mathbf{B}}++0_{1 ; 0}^{+} \cdot \breve{\boldsymbol{\phi}}_{p_{\bullet}+2 ; 2(p \bullet+1)}-\left(p_{\bullet}+3\right) \stackrel{\circ}{\mathbf{B}}_{1 ; 0}^{-} \cdot \breve{\boldsymbol{\phi}}_{p_{\bullet}+2 ; 2(p \bullet+1)} \\
& -\frac{1}{2}\left(p_{\bullet}+3\right)\left(p_{\bullet}+2\right) \stackrel{\circ}{\mathbf{B}}_{2 ; 0} \cdot \breve{\boldsymbol{\phi}}_{p_{\bullet}+1 ; 2\left(p_{\bullet}+1\right)}^{\prime}-\frac{1}{2}\left(p_{\bullet}+3\right)\left(p_{\bullet}+2\right) \stackrel{\circ}{\mathbf{B}}_{2 ; 0}^{+} \cdot \breve{\boldsymbol{\phi}}_{p_{\bullet}+1 ; 2(p \bullet+1)} \\
& -\frac{1}{2}\left(p_{\bullet}+3\right)\left(p_{\bullet}+2\right) \stackrel{\circ}{\mathbf{B}}_{2 ; 0}^{-} \cdot \breve{\boldsymbol{\phi}}_{p_{\bullet}+1 ; 2\left(p_{\bullet}+1\right)}-\left(p_{\bullet}+3\right) \breve{\mathbf{B}}_{p_{\bullet}+2 ; 2(p \bullet+1)} \cdot \stackrel{\circ}{\boldsymbol{\phi}}_{1 ; 0}^{\prime}
\end{aligned}
$$


As in the analysis of lower order transport equations, we begin by noticing that the entries of

$$
\stackrel{\circ}{\boldsymbol{v}}_{0 ; 0}, \quad \stackrel{\circ}{\mathbf{L}}_{1 ; 0}, \quad \stackrel{\circ}{\mathbf{F}}_{0 ; 0}, \quad \stackrel{\circ}{\mathbf{G}}_{0 ; 0}
$$

are Minkowskian -i.e. independent of $m$ and $W$. On the other hand, the entries of

$$
\begin{array}{cccccc}
\stackrel{\circ}{\boldsymbol{v}}_{1 ; 0}, & \stackrel{\circ}{\mathbf{L}}_{2 ; 0}, & \stackrel{\circ}{\phi}_{0 ; 0}, & \stackrel{\circ}{\phi}_{1 ; 0}, & & \\
\stackrel{\circ}{\mathbf{F}}_{1 ; 0}, & \stackrel{\circ}{\mathbf{F}}_{2 ; 0}, & \stackrel{\circ}{\mathbf{A}}_{1 ; 0}, & \stackrel{\circ}{\mathbf{A}_{1 ; 0}^{ \pm},} & \stackrel{\circ}{\mathbf{A}}_{2 ; 0}, & \stackrel{\circ}{\mathbf{A}}_{2 ; 0}^{ \pm}, \\
\stackrel{\circ}{\mathbf{G}}_{1 ; 0}, & \stackrel{\circ}{\mathbf{G}}_{2 ; 0}, & \stackrel{\circ}{\mathbf{B}}_{1 ; 0}, & \stackrel{\circ}{\mathbf{B}}_{1 ; 0}^{ \pm}, & \stackrel{\circ}{\mathbf{B}}_{2 ; 0}, & \stackrel{\circ}{\mathbf{B}}_{2 ; 0}^{ \pm},
\end{array}
$$

are Schwarzschildean in our gauge - i.e. they depend only on $m$.

As a consequence of the analysis of the transport equations for the orders $p_{\bullet}+1$ and $p_{\bullet}+2$ one has that the entries in

$$
\breve{\boldsymbol{v}}_{p_{\bullet}+1 ; 2\left(p_{\bullet}+1\right)}, \quad \breve{\boldsymbol{\phi}}_{p_{\bullet}+1 ; 2\left(p_{\bullet}+1\right)}, \quad \breve{\boldsymbol{\phi}}_{p_{\bullet}+2 ; 2(p \bullet+1)},
$$

depend only on $m$ and the coefficients $\breve{w}_{p_{\bullet}+1 ; 2\left(p_{\bullet}+1\right) ; k}$. As in the analysis of the orders $p_{\bullet}+1$ and $p_{\bullet}+2$ one has the crucial observation:

Observation 3. The equations (24a) $-(24 \mathrm{c})$ and their initial conditions are formally identical to the order $p_{\bullet}+3$ transport equations for initial data sets which are Schwarzschildean up to order $p_{\bullet}$.

As in the analysis of the orders $p_{\bullet}+1, p_{\bullet}+2$ and $p_{\bullet}+3$, one can use directly the analysis in 22] to conclude that the solutions $\dot{\boldsymbol{v}}_{p_{\bullet}+3 ; 2\left(p_{\bullet}+1\right)}$ and $\dot{\phi}_{p_{\bullet}+3 ; 2\left(p_{\bullet}+1\right)}$ to equations (24a)-(24c) are polynomial in $\tau$. More generally, one has that:

Proposition 5. The solutions to the order $p_{\bullet}+3$ transport equations (18a) $-(18 \mathrm{c}$ ) are polynomial in $\tau$ and, thus, extend analytically through $\tau= \pm 1$.

\subsection{The transport equations at order $p=p_{\bullet}+4$}

Finally, using similar methods, we discuss the order $p_{\bullet}+4$ transport equations. As in the previous order the polynomial dependence in $\tau$ follows directly from Lemma 4 once one knows that the entries in $\phi^{\left(p_{\bullet}+3\right)}$ are polynomial. The rest of the analysis is more subtle than at lower orders. The reason for this is twofold:

(i) As a consequence of Lemma 6 one finds that $\breve{\phi}_{A B C D}^{(p \bullet+3)}$ has a multipolar structure which is more complicated than that of data which just deviates from Schwarzschild data.

(ii) The discussion of the transport equations for the sectors in $\mathfrak{S}_{p_{\bullet}+1}$ involves non-trivial multiplications of the functions $T_{i k}^{j}$.

Evidence from explicit calculations suggests that the solutions to the Bianchi transport equations at this order are, generically, not smooth at the critical sets.

Proceeding as in the case of lower order one finds that the substitution of the Ansätze

$$
\boldsymbol{v}^{(p)}=\stackrel{\circ}{\boldsymbol{v}}^{(p)}+\breve{\boldsymbol{v}}^{(p)}, \quad \boldsymbol{\phi}^{(p)}=\stackrel{\circ}{\boldsymbol{\phi}}^{(p)}+\breve{\boldsymbol{\phi}}^{(p)}, \quad 0 \leq p \leq p_{\bullet}+4
$$

into the transport equations (18a)-(18c) and taking into account Lemma 8 leads to the following equations for $\breve{\boldsymbol{v}}^{\left(p_{\bullet}+4\right)}$ and $\breve{\boldsymbol{\phi}}^{\left(p_{\bullet}+4\right)}$ :

$$
\begin{aligned}
& \partial_{\tau} \breve{\boldsymbol{v}}^{(p \bullet+4)}=\mathbf{K} \cdot \breve{\boldsymbol{v}}^{(p \bullet+4)}+\mathbf{Q}\left(\stackrel{\boldsymbol{v}}{ }^{(0)}, \breve{\boldsymbol{v}}^{(p \bullet+4)}\right)+\mathbf{Q}\left(\breve{\boldsymbol{v}}^{(p \bullet+4)}, \stackrel{\boldsymbol{v}}{(0)}^{(0)}\right. \\
& +\left(p_{\bullet}+4\right)\left(\mathbf{Q}\left(\dot{\boldsymbol{v}}^{(1)}, \breve{\boldsymbol{v}}^{\left(p_{\bullet}+3\right)}\right)+\mathbf{Q}\left(\breve{\boldsymbol{v}}^{\left(p_{\bullet}+3\right)}, \dot{\boldsymbol{v}}^{(1)}\right)\right)
\end{aligned}
$$

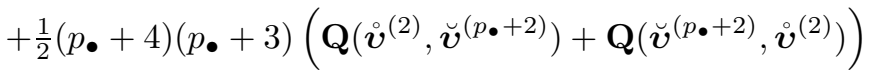

$$
\begin{aligned}
& +\breve{\mathbf{L}}^{\left(p_{\bullet}+4\right)} \cdot \stackrel{\circ}{\phi}^{(0)}+\left(p_{\bullet}+4\right) \breve{\mathbf{L}}^{(p \bullet+3)} \cdot \stackrel{\circ}{\phi}^{(1)} \\
& +\left(p_{\bullet}+4\right) \stackrel{\circ}{\mathbf{L}}^{(1)} \cdot \breve{\boldsymbol{\phi}}^{\left(p_{\bullet}+3\right)}+\frac{1}{2}\left(p_{\bullet}+4\right)\left(p_{\bullet}+3\right) \stackrel{\circ}{\mathbf{L}}^{(2)} \cdot \breve{\boldsymbol{\phi}}^{\left(p_{\bullet}+2\right)} \\
& +\frac{1}{6}\left(p_{\bullet}+4\right)\left(p_{\bullet}+3\right)\left(p_{\bullet}+2\right) \stackrel{\circ}{\mathbf{L}}^{(3)} \cdot \dot{\phi}^{\left(p_{\bullet}+1\right)},
\end{aligned}
$$




$$
\begin{aligned}
& \sqrt{2} \mathbf{E} \partial_{\tau} \breve{\phi}^{\left(p_{\bullet}+4\right)}+\left(\stackrel{\AA}{\mathbf{A}}^{\mu}\right)^{(0)} \partial_{\mu} \breve{\boldsymbol{\phi}}^{\left(p_{\bullet}+4\right)}=\stackrel{\circ}{\mathbf{F}}^{(0)} \cdot \breve{\boldsymbol{\phi}}_{p_{\bullet}+4}+\left(p_{\bullet}+4\right) \stackrel{\circ}{\mathbf{F}}^{(1)} \cdot \breve{\boldsymbol{\phi}}^{\left(\boldsymbol{\bullet}_{\bullet}+3\right)} \\
& +\frac{1}{2}\left(p_{\bullet}+4\right)\left(p_{\bullet}+4\right) \stackrel{\circ}{\mathbf{F}}^{(2)} \cdot \breve{\boldsymbol{\phi}}^{\left(p_{\bullet}+2\right)}+\frac{1}{6}\left(p_{\bullet}+4\right)\left(p_{\bullet}+3\right)\left(p_{\bullet}+2\right) \stackrel{\circ}{\mathbf{F}}^{(3)} \cdot \breve{\boldsymbol{\phi}}^{\left(p_{\bullet}+1\right)} \\
& +\frac{1}{2}\left(p_{\bullet}+4\right)\left(p_{\bullet}+3\right) \breve{\mathbf{F}}^{\left(p_{\bullet}+2\right)} \cdot \stackrel{\circ}{\boldsymbol{\phi}}^{(2)}+\left(p_{\bullet}+4\right) \breve{\mathbf{F}}^{\left(p_{\bullet}+3\right)} \cdot \stackrel{\circ}{\boldsymbol{\phi}}^{(1)}+\breve{\mathbf{F}}^{\left(p_{\bullet}+4\right)} \cdot \stackrel{\circ}{\boldsymbol{\phi}}^{(0)} \\
& -\left(p_{\bullet}+4\right)\left(\AA^{\mu}\right)^{(1)} \partial_{\mu} \breve{\boldsymbol{\phi}}^{\left(\boldsymbol{\bullet}_{\bullet}+3\right)}-\frac{1}{2}\left(p_{\bullet}+4\right)\left(p_{\bullet}+3\right)\left(\AA^{\mu}\right)^{(2)} \partial_{\mu} \breve{\boldsymbol{\phi}}^{\left(p_{\bullet}+2\right)} \\
& -\frac{1}{6}\left(p_{\bullet}+4\right)\left(p_{\bullet}+3\right)\left(p_{\bullet}+2\right)\left(\AA^{\mu}\right)^{(3)} \partial_{\mu} \breve{\boldsymbol{\phi}}^{\left(p_{\bullet}+1\right)} \\
& -\frac{1}{2}\left(p_{\bullet}+4\right)\left(p_{\bullet}+3\right)\left(\breve{\mathbf{A}}^{\mu}\right)^{\left(p_{\bullet}+2\right)} \partial_{\mu} \stackrel{\circ}{\boldsymbol{\phi}}(2)^{(2)}\left(p_{\bullet}+4\right)\left(\breve{\mathbf{A}}^{\mu}\right)^{\left(p_{\bullet}+3\right)} \partial_{\mu} \stackrel{\circ}{\boldsymbol{\phi}}^{(1)}-\left(\breve{\mathbf{A}}^{\mu}\right)^{\left(p_{\bullet}+4\right)} \partial_{\mu} \stackrel{\circ}{(0)}^{(0)}
\end{aligned}
$$

$$
\begin{aligned}
& \left(\stackrel{\circ}{ }^{\mu}\right)^{(0)} \partial_{\mu} \breve{\boldsymbol{\phi}}^{\left(p_{\bullet}+4\right)}=\stackrel{\circ}{\mathbf{G}}^{(0)} \cdot \breve{\boldsymbol{\phi}}_{p_{\bullet}+4}+\left(p_{\bullet}+4\right) \stackrel{\circ}{\mathbf{G}}^{(1)} \cdot \breve{\boldsymbol{\phi}}^{\left(p_{\bullet}+3\right)} \\
& +\frac{1}{2}\left(p_{\bullet}+4\right)\left(p_{\bullet}+3\right) \stackrel{\circ}{\mathbf{G}}^{(2)} \cdot \breve{\boldsymbol{\phi}}^{\left(p_{\bullet}+2\right)}+\frac{1}{6}\left(p_{\bullet}+4\right)\left(p_{\bullet}+3\right)\left(p_{\bullet}+2\right) \stackrel{\circ}{\mathbf{G}}^{(3)} \cdot \breve{\boldsymbol{\phi}}^{\left(p_{\bullet}+1\right)} \\
& +\frac{1}{2}\left(p_{\bullet}+4\right)\left(p_{\bullet}+3\right) \breve{\mathbf{G}}^{\left(p_{\bullet}+2\right)} \cdot \stackrel{\circ}{\boldsymbol{\phi}}^{(2)}+\left(p_{\bullet}+4\right) \breve{\mathbf{G}}^{\left(p_{\bullet}+3\right)} \cdot \stackrel{\circ}{\boldsymbol{\phi}}^{(1)}+\breve{\mathbf{G}}^{\left(p_{\bullet}+4\right)} \cdot \stackrel{\circ}{\boldsymbol{\phi}}^{(0)} \\
& -\left(p_{\bullet}+4\right)\left(\stackrel{\circ}{\mathbf{B}}^{\mu}\right)^{(1)} \partial_{\mu} \breve{\boldsymbol{\phi}}^{\left(p_{\bullet}+3\right)}-\frac{1}{2}\left(p_{\bullet}+4\right)\left(p_{\bullet}+3\right)\left(\stackrel{\mathrm{B}}{ }^{\mu}\right)^{(2)} \partial_{\mu} \breve{\boldsymbol{\phi}}^{\left(p_{\bullet}+2\right)} \\
& -\frac{1}{6}\left(p_{\bullet}+4\right)\left(p_{\bullet}+3\right)\left(p_{\bullet}+2\right)\left(\stackrel{\circ}{ }^{\mu}\right)^{(3)} \partial_{\mu} \breve{\boldsymbol{\phi}}^{\left(p_{\bullet}+1\right)} \\
& -\frac{1}{2}\left(p_{\bullet}+4\right)\left(p_{\bullet}+3\right)\left(\breve{\mathbf{B}}^{\mu}\right)^{\left(\boldsymbol{p}_{\bullet}+2\right)} \partial_{\mu} \stackrel{\circ}{\boldsymbol{\phi}}^{(2)}-\left(p_{\bullet}+4\right)\left(\breve{\mathbf{B}}^{\mu}\right)^{\left(p_{\bullet}+3\right)} \partial_{\mu} \stackrel{\circ}{\boldsymbol{\phi}}^{(1)}-\left(\breve{\mathbf{B}}^{\mu}\right)^{\left(p_{\bullet}+4\right)} \partial_{\mu} \stackrel{\circ}{\boldsymbol{\phi}}(0)^{(0)}
\end{aligned}
$$

As in the previous orders, we extract equations for the components of $\mathfrak{S}_{p_{\bullet}+1}\left[\boldsymbol{v}^{\left(p_{\bullet}+4\right)}, \boldsymbol{\phi}^{\left(p_{\bullet}+4\right)}\right]$. In this case the analysis is more involved as there are terms involving non-trivial products of spherical harmonics. An inspection shows that the terms containing this type of non-trivial products are

$$
\begin{aligned}
& \stackrel{\circ}{\mathbf{F}}^{(3)} \cdot \breve{\boldsymbol{\phi}}^{\left(p_{\bullet}+1\right)}, \quad \breve{\mathbf{F}}^{\left(p_{\bullet}+2\right)} \cdot \stackrel{\circ}{\boldsymbol{\phi}}^{(2)}, \quad\left(\stackrel{\AA}{\mathbf{A}}^{\mu}\right)^{(3)} \cdot \partial_{\mu} \breve{\boldsymbol{\phi}}^{\left(p_{\bullet}+1\right)}, \quad\left(\breve{\mathbf{A}}^{\mu}\right)^{\left(p_{\bullet}+2\right)} \cdot \partial_{\mu} \breve{\boldsymbol{\phi}}^{(2)}, \\
& \stackrel{\mathbf{G}}{ }^{(3)} \cdot \breve{\boldsymbol{\phi}}^{\left(p_{\bullet}+1\right)}, \quad \breve{\mathbf{G}}^{\left(p_{\bullet}+2\right)} \cdot \stackrel{\circ}{\boldsymbol{\phi}}^{(2)}, \quad\left(\stackrel{\circ}{\mathbf{B}}^{\mu}\right)^{(3)} \cdot \partial_{\mu} \breve{\boldsymbol{\phi}}^{\left(p_{\bullet}+1\right)}, \quad\left(\breve{\mathbf{B}}^{\mu}\right)^{\left(p_{\bullet}+2\right)} \cdot \partial_{\mu} \breve{\boldsymbol{\phi}}^{(2)} .
\end{aligned}
$$

All these terms contain products of the form $T_{4}{ }^{j_{1}} k_{1} \times T_{2\left(p_{\bullet}+1\right)}{ }^{i_{2}}{ }_{j_{2}}$, which using formula (9) can be linearised to render

$$
\begin{aligned}
& T_{4}{ }^{j_{1}} k_{1} \times T_{2\left(p_{\bullet}+1\right)}{ }^{i_{2}} j_{2} \\
& =c_{2\left(p_{\bullet}+3\right), j_{1}, j_{2}, k_{1}, k_{2}} T_{2\left(p_{\bullet}+3\right)}{ }_{k_{1}+j_{2}}^{j_{1}+k_{2}}+c_{2(p \bullet+1), j_{1}, j_{2}, k_{1}, k_{2}} T_{2\left(p_{\bullet}+1\right)}^{j_{1}+j_{2}-1} k_{1}+k_{2}-1
\end{aligned}
$$

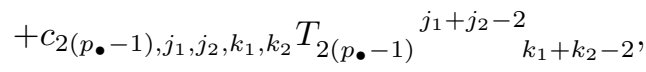

with $c_{2\left(p_{\bullet}+3\right), j_{1}, j_{2}, k_{1}, k_{2}}, c_{2\left(p_{\bullet}+1\right), j_{1}, j_{2}, k_{1}, k_{2}}, c_{2\left(p_{\bullet}-1\right), j_{1}, j_{2}, k_{1}, k_{2}}$ some numerical coefficients. Their explicit form will not be essential for the subsequent analysis. Thus, the sector $\mathfrak{S}_{p_{\bullet}+1}$ of, say, $\stackrel{\circ}{\mathbf{F}}^{(3)} \cdot \breve{\boldsymbol{\phi}}^{\left(p_{\bullet}+1\right)}$ is of the form

$$
\stackrel{\circ}{\mathbf{F}}_{3 ; 0} \cdot \breve{\boldsymbol{\phi}}_{p_{\bullet}+1 ; 2\left(p_{\bullet}+1\right)}+\mathbf{C}_{4,2\left(p_{\bullet}+1\right) ; p_{\bullet}+1} \cdot \stackrel{\circ}{\mathbf{F}}_{3 ; 4} \cdot \breve{\boldsymbol{\phi}}_{p_{\bullet}+1 ; 2\left(p_{\bullet}+1\right)},
$$

where $\mathbf{C}_{4,2(p \bullet+1) ; p_{\bullet}+1}$ denotes a matrix with numerical entries. The term $\stackrel{\circ}{\mathbf{F}}_{3 ; 0} \cdot \breve{\boldsymbol{\phi}}_{p_{\bullet}+1 ; 2(p \bullet+1)}$ is formally identical to the one one would obtain from considering asymptotically Schwarzschildean data. A similar analysis can be carried out with the other terms containing non-trivial products.

The equations for the components of $\mathfrak{S}_{p_{\bullet}+1}\left[\breve{\boldsymbol{v}}^{\left(p_{\bullet}+4\right)}, \breve{\boldsymbol{\phi}}^{\left(p_{\bullet}+4\right)}\right]$ are:

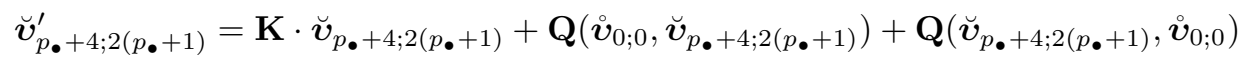

$$
\begin{aligned}
& +\left(p_{\bullet}+4\right)\left(\mathbf{Q}\left(\dot{\boldsymbol{v}}_{1 ; 0}, \breve{\boldsymbol{v}}_{p_{\bullet}+3 ; 2\left(p_{\bullet}+1\right)}\right)+\mathbf{Q}\left(\breve{\boldsymbol{v}}_{p_{\bullet}+3 ; 2\left(p_{\bullet}+1\right)}, \stackrel{\boldsymbol{v}}{1 ; 0}_{1 ; 0}\right)\right) \\
& +\frac{1}{2}\left(p_{\bullet}+4\right)\left(p_{\bullet}+3\right)\left(\mathbf{Q}\left(\stackrel{\boldsymbol{v}}{2 ; 0}_{2}, \breve{\boldsymbol{v}}_{p_{\bullet}+2 ; 2\left(p_{\bullet}+1\right)}\right)+\mathbf{Q}\left(\breve{\boldsymbol{v}}_{p_{\bullet}+2 ; 2\left(p_{\bullet}+1\right)}, \stackrel{\circ}{2 ; 0}_{2}\right)\right) \\
& +\left(p_{\bullet}+4\right) \stackrel{\circ}{\mathbf{L}}_{1 ; 0} \cdot \breve{\boldsymbol{\phi}}_{p_{\bullet}+3 ; 2(p \bullet+1)}+\frac{1}{2}\left(p_{\bullet}+4\right)\left(p_{\bullet}+3\right) \stackrel{\circ}{\mathbf{L}}_{2 ; 0} \cdot \breve{\boldsymbol{\phi}}_{p_{\bullet}+2 ; 2(p \bullet+1)} \\
& +\frac{1}{6}\left(p_{\bullet}+4\right)\left(p_{\bullet}+3\right)\left(p_{\bullet}+2\right) \stackrel{\circ}{\mathbf{L}}_{3 ; 0} \cdot \breve{\phi}_{p_{\bullet}+1 ; 2\left(p_{\bullet}+1\right)} \\
& +\stackrel{\circ}{\mathbf{L}}_{p_{\bullet}+4 ; 2\left(p_{\bullet}+1\right)} \cdot \stackrel{\circ}{\phi}_{0 ; 0}+(p \bullet+4) \stackrel{\circ}{\mathbf{L}}_{p_{\bullet}+3 ; 2\left(p_{\bullet}+1\right)} \cdot \stackrel{\circ}{\phi}_{1 ; 0},
\end{aligned}
$$




$$
\begin{aligned}
& (\mathbf{E}+\mathbf{A}) \cdot \breve{\boldsymbol{\phi}}_{p_{\bullet}+4 ; 2\left(p_{\bullet}+1\right)}^{\prime}+\mathbf{A}_{p_{\bullet}+4 ; 2\left(p_{\bullet}+1\right)} \cdot \breve{\boldsymbol{\phi}}_{p_{\bullet}+4 ; 2\left(p_{\bullet}+1\right)}={\stackrel{\circ}{\mathbf{F}_{0 ; 0}}}_{0} \breve{\boldsymbol{\phi}}_{p_{\bullet}+4 ; 2\left(p_{\bullet}+1\right)}+\left(p_{\bullet}+4\right) \stackrel{\circ}{\mathbf{F}}_{1 ; 0} \cdot \breve{\boldsymbol{\phi}}_{p_{\bullet}+3 ; 2\left(p_{\bullet}+1\right)} \\
& +\frac{1}{2}\left(p_{\bullet}+4\right)\left(p_{\bullet}+3\right) \stackrel{\circ}{\mathbf{F}}_{2 ; 0} \cdot \breve{\boldsymbol{\phi}}_{p_{\bullet}+2 ; 2(p \bullet+1)}+\frac{1}{6}\left(p_{\bullet}+4\right)(p \bullet+3)\left(p_{\bullet}+2\right) \stackrel{\circ}{\mathbf{F}}_{3 ; 0} \cdot \breve{\phi}_{p_{\bullet}+1 ; 2\left(p_{\bullet}+1\right)} \\
& +\breve{\mathbf{F}}_{p_{\bullet}+4 ; 2(p \bullet+1)} \cdot \stackrel{\circ}{0 ; 0}_{0}+\left(p_{\bullet}+4\right) \breve{\mathbf{F}}_{p_{\bullet}+3 ; 2(p \bullet+1)} \cdot \stackrel{\circ}{1 ; 0}_{1 ;}+\frac{1}{2}\left(p_{\bullet}+4\right)\left(p_{\bullet}+3\right) \breve{\mathbf{F}}_{p \bullet+2 ; 2(p \bullet+1)} \cdot \stackrel{\circ}{\phi}_{2 ; 0}
\end{aligned}
$$

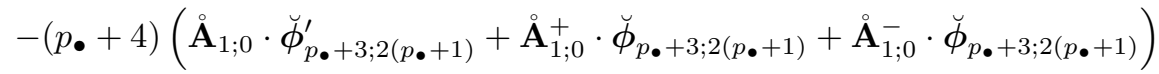

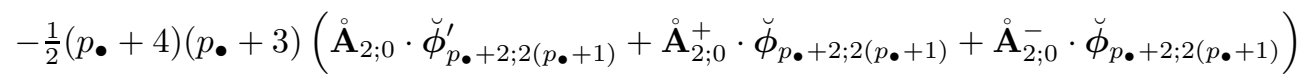

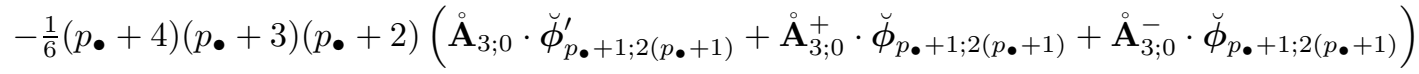

$$
\begin{aligned}
& -\left(p_{\bullet}+4\right) \breve{\mathbf{A}}_{p_{\bullet}+3 ; 2\left(p_{\bullet}+1\right)} \cdot \stackrel{\circ}{1 ; 0}^{\prime}-\frac{1}{2}\left(p_{\bullet}+4\right)\left(p_{\bullet}+3\right) \breve{\mathbf{A}}_{p \bullet+2 ; 2\left(p_{\bullet}+1\right)} \cdot \stackrel{\circ}{\phi}_{2 ; 0}^{\prime} \\
& +\mathbf{R}_{2\left(p_{\bullet}+1\right) ; p_{\bullet}+1} \text {, }
\end{aligned}
$$

$\mathbf{B} \cdot \breve{\boldsymbol{\phi}}_{p_{\bullet}+4 ; 2\left(p_{\bullet}+1\right)}^{\prime}+\mathbf{B}_{p_{\bullet}+4 ; 2\left(p_{\bullet}+1\right)} \cdot \breve{\boldsymbol{\phi}}_{p_{\bullet}+4 ; 2(p \bullet+1)}=\stackrel{\circ}{\mathbf{G}}_{0 ; 0} \cdot \breve{\boldsymbol{\phi}}_{p_{\bullet}+4 ; 2\left(p_{\bullet}+1\right)}+\left(p_{\bullet}+4\right) \stackrel{\circ}{\mathbf{G}}_{1 ; 0} \cdot \breve{\boldsymbol{\phi}}_{p_{\bullet}+3 ; 2(p \bullet+1)}$

$+\frac{1}{2}\left(p_{\bullet}+4\right)\left(p_{\bullet}+3\right) \stackrel{\circ}{\mathbf{G}}_{2 ; 0} \cdot \breve{\boldsymbol{\phi}}_{p_{\bullet}+2 ; 2\left(p_{\bullet}+1\right)}+\frac{1}{6}\left(p_{\bullet}+4\right)\left(p_{\bullet}+3\right)\left(p_{\bullet}+2\right) \stackrel{\circ}{\mathbf{G}}_{3 ; 0} \cdot \breve{\phi}_{p_{\bullet}+1 ; 2\left(p_{\bullet}+1\right)}$

$+\breve{\mathbf{G}}_{p_{\bullet}+4 ; 2\left(p_{\bullet}+1\right)} \cdot \stackrel{\circ}{0 ; 0}_{0}+\left(p_{\bullet}+4\right) \breve{\mathbf{G}}_{p_{\bullet}+3 ; 2\left(p_{\bullet}+1\right)} \cdot \stackrel{\circ}{\phi}_{1 ; 0}+\frac{1}{2}\left(p_{\bullet}+4\right)(p \bullet+3) \breve{\mathbf{G}}_{p_{\bullet}+2 ; 2(p \bullet+1)} \cdot \stackrel{\circ}{\phi}_{2 ; 0}$

$-\left(p_{\bullet}+4\right)\left(\stackrel{\circ}{\mathbf{B}}_{1 ; 0} \cdot \breve{\boldsymbol{\phi}}_{p_{\bullet}+3 ; 2\left(p_{\bullet}+1\right)}^{\prime}+\stackrel{\circ}{\mathbf{B}}+1 ; 0^{+} \cdot \breve{\boldsymbol{\phi}}_{p_{\bullet}+3 ; 2\left(p_{\bullet}+1\right)}+\stackrel{\circ}{\mathbf{B}}_{1 ; 0}^{-} \cdot \breve{\boldsymbol{\phi}}_{p_{\bullet}+3 ; 2\left(p_{\bullet}+1\right)}\right)$

$-\frac{1}{2}\left(p_{\bullet}+4\right)(p \bullet+3)\left(\stackrel{\circ}{\mathbf{B}}_{2 ; 0} \cdot \breve{\boldsymbol{\phi}}_{p_{\bullet}+2 ; 2\left(p_{\bullet}+1\right)}^{\prime}+\stackrel{\circ}{\mathbf{B}}+; 0^{+} \cdot \breve{\boldsymbol{\phi}}_{p_{\bullet}+2 ; 2(p \bullet+1)}+\stackrel{\circ}{\mathbf{B}}_{2 ; 0}^{-} \cdot \breve{\boldsymbol{\phi}}_{p_{\bullet}+2 ; 2(p \bullet+1)}\right)$

$-\frac{1}{6}\left(p_{\bullet}+4\right)\left(p_{\bullet}+3\right)\left(p_{\bullet}+2\right)\left(\stackrel{\circ}{\mathbf{B}}_{3 ; 0} \cdot \breve{\boldsymbol{\phi}}_{p_{\bullet}+1 ; 2\left(p_{\bullet}+1\right)}^{\prime}+\stackrel{\circ}{\mathbf{B}}_{3 ; 0}^{+} \cdot \breve{\boldsymbol{\phi}}_{p_{\bullet}+1 ; 2\left(p_{\bullet}+1\right)}+\stackrel{\circ}{\mathbf{B}}_{3 ; 0}^{-} \cdot \breve{\boldsymbol{\phi}}_{p_{\bullet}+1 ; 2\left(p_{\bullet}+1\right)}\right)$

$-\left(p_{\bullet}+4\right) \breve{\mathbf{B}}_{p_{\bullet}+3 ; 2\left(p_{\bullet}+1\right)} \cdot \stackrel{\circ}{1 ; 0}^{\prime}-\frac{1}{2}\left(p_{\bullet}+4\right)\left(p_{\bullet}+3\right) \breve{\mathbf{B}}_{p_{\bullet}+2 ; 2\left(p_{\bullet}+1\right)} \cdot{\stackrel{\circ}{\phi_{2}^{\prime} ; 0}}^{\prime}$

$+\mathbf{S}_{2\left(p_{\bullet}+1\right) ; p_{\bullet}+1}$

where

$$
\begin{aligned}
& \mathbf{R}_{2\left(p_{\bullet}+1\right) ; p_{\bullet}+1}=\left(p_{\bullet}+4\right)\left(p_{\bullet}+3\right) \mathbf{C}_{4,2\left(p_{\bullet}+1\right) ; p_{\bullet}+1} \cdot\left[\frac{1}{6}\left(p_{\bullet}+2\right) \stackrel{\circ}{\mathbf{F}}_{3 ; 4} \cdot \breve{\phi}_{p_{\bullet}+1 ; 2(p \bullet+1)}+\frac{1}{2} \breve{\mathbf{F}}_{p \bullet+2 ; 2(p \bullet+1)} \cdot \stackrel{\circ}{\phi}_{2 ; 4}\right. \\
& -\frac{1}{6}(p \bullet+2)\left(\stackrel{\AA}{\mathbf{A}}_{3 ; 4} \cdot \breve{\boldsymbol{\phi}}_{p_{\bullet}+1 ; 2(p \bullet+1)}^{\prime}+\stackrel{\AA}{\mathbf{A}}_{3 ; 4}^{+} \cdot \breve{\boldsymbol{\phi}}_{p_{\bullet}+1 ; 2(p \bullet+1)}+\stackrel{\AA}{\mathbf{A}}_{3 ; 4}^{-} \cdot \breve{\boldsymbol{\phi}}_{p_{\bullet}+1 ; 2\left(p_{\bullet}+1\right)}\right) \\
& \left.-\frac{1}{6}\left(p_{\bullet}+2\right)\left(\breve{\mathbf{A}}_{p_{\bullet}+2 ; 2\left(p_{\bullet}+1\right)} \cdot \stackrel{\circ}{2 ; 4}_{2}^{\prime}+\breve{\mathbf{A}}_{p_{\bullet}+2 ; 2\left(p_{\bullet}+1\right)}^{+} \cdot \stackrel{\circ}{\phi}_{2 ; 4}+\breve{\mathbf{A}}_{p_{\bullet}+2 ; 2\left(p_{\bullet}+1\right)}^{-} \cdot \stackrel{\circ}{\phi}_{2 ; 4}\right)\right] \\
& \mathbf{S}_{2\left(p_{\bullet}+1\right) ; p_{\bullet}+1}=\left(p_{\bullet}+4\right)(p \bullet+3) \mathbf{C}_{4,2\left(p_{\bullet}+1\right) ; p_{\bullet}+1} \cdot\left[\frac{1}{6}(p \bullet+2) \stackrel{\circ}{\mathbf{G}}_{3 ; 4} \cdot \breve{\phi}_{p_{\bullet}+1 ; 2(p \bullet+1)}+\frac{1}{2} \breve{\mathbf{G}}_{p_{\bullet}+2 ; 2(p \bullet+1)} \cdot \stackrel{\circ}{2 ; 4}\right. \\
& -\frac{1}{6}(p \bullet+2)\left(\stackrel{\circ}{\mathbf{B}}_{3 ; 4} \cdot \breve{\phi}_{p_{\bullet}+1 ; 2(p \bullet+1)}^{\prime}+\stackrel{\circ}{\mathbf{B}}_{3 ; 4}^{+} \cdot \breve{\boldsymbol{\phi}}_{p \bullet+1 ; 2(p \bullet+1)}+\stackrel{\circ}{\mathbf{B}}_{3 ; 4}^{-} \cdot \breve{\boldsymbol{\phi}}_{p_{\bullet}+1 ; 2(p \bullet+1)}\right) \\
& \left.-\frac{1}{6}\left(p_{\bullet}+2\right)\left(\breve{\mathbf{B}}_{p_{\bullet}+2 ; 2\left(p_{\bullet}+1\right)} \cdot \stackrel{\circ}{\phi}_{2 ; 4}^{\prime}+\breve{\mathbf{B}}_{p_{\bullet}+2 ; 2\left(p_{\bullet}+1\right)}^{+} \cdot \stackrel{\circ}{\phi}_{2 ; 4}+\breve{\mathbf{B}}_{p_{\bullet}+2 ; 2\left(p_{\bullet}+1\right)}^{-} \cdot \stackrel{\circ}{\phi}_{2 ; 4}\right)\right] .
\end{aligned}
$$

The initial data for the transport equations (25b)-(25c) can be written as

$$
\breve{\phi}_{p_{\bullet}+4,2\left(p_{\bullet}+1\right)}(0)=\tilde{\boldsymbol{\phi}}_{p_{\bullet}+4,2\left(p_{\bullet}+1\right)}(0)+\hat{\boldsymbol{\phi}}_{p_{\bullet}+4,2\left(p_{\bullet}+1\right)}(0),
$$

where $\tilde{\boldsymbol{\phi}}_{p_{\bullet}+4,2\left(p_{\bullet}+1\right)}$ depends solely on $m$ and $\breve{w}_{p_{\bullet}+1 ; 2\left(p_{\bullet}+1\right), k}$ (deviation from Schwarzschild) while $\hat{\boldsymbol{\phi}}_{\boldsymbol{\bullet}_{\bullet}+4,2\left(p_{\bullet}+1\right)}$ contains contributions from the multipolar structure of the reference static data.

In order to discuss equations (25a)-(25c) we introduce a further Ansatz. We write

$$
\begin{aligned}
& \breve{\boldsymbol{v}}_{p_{\bullet}+4,2\left(p_{\bullet}+1\right)}=\tilde{\boldsymbol{v}}_{p_{\bullet}+4,2\left(p_{\bullet}+1\right)}+\hat{\boldsymbol{v}}_{p_{\bullet}+4,2\left(p_{\bullet}+1\right)}, \\
& \breve{\boldsymbol{\phi}}_{p_{\bullet}+4,2\left(p_{\bullet}+1\right)}=\tilde{\boldsymbol{\phi}}_{p_{\bullet}+4,2\left(p_{\bullet}+1\right)}+\hat{\boldsymbol{\phi}}_{p_{\bullet}+4,2\left(p_{\bullet}+1\right)},
\end{aligned}
$$

where $\tilde{\boldsymbol{v}}_{p_{\bullet}+4,2\left(p_{\bullet}+1\right)}$ and $\tilde{\boldsymbol{v}}_{p_{\bullet}+4,2\left(p_{\bullet}+1\right)}$ are the solutions to equations (25a)-(25c) with

$$
\mathbf{R}_{2\left(p_{\bullet}+1\right) ; p_{\bullet}+1}=0, \quad \mathbf{S}_{2\left(p_{\bullet}+1\right) ; p_{\bullet}+1}=0, \quad \hat{\boldsymbol{\phi}}_{p_{\bullet}+4 ; 2\left(p_{\bullet}+1\right)}(0)=0 .
$$

The crucial observation is the following: 
Observation 4. Equations (25a)-(25c) and the data (27) satisfying (29) are formally identical to the order $p_{\bullet}+4$ transport equations analysed in [22] for data which are Schwarzschildean up to order $p_{\bullet}$. These equations have no solution which is smooth at the critical sets.

From the analysis in 22 it follows that:

$$
\begin{aligned}
\partial_{\tau}^{11} \tilde{\boldsymbol{\phi}}_{p_{\bullet}+4 ; 2(p \bullet+1)}=m^{3} \breve{w}_{p_{\bullet}}+1 ; 2(p \bullet+1) & (1-\tau)^{p \bullet-15}(1+\tau)^{p \bullet-15} \\
& \times\left(\tilde{\boldsymbol{\varphi}}_{p_{\bullet}+4}(\tau)+\tilde{\boldsymbol{\varphi}}_{p_{\bullet}+4}^{+}(\tau) \ln (1+\tau)+\tilde{\boldsymbol{\varphi}}_{p_{\bullet}+4}^{-}(\tau) \ln (1-\tau)\right)
\end{aligned}
$$

where the entries of $\tilde{\varphi}, \tilde{\varphi}^{ \pm}$are polynomials of degree 33. Substitution of the Ansatz (28a $)$-(28b) into equations (25b)-(25c) renders:

$$
\begin{aligned}
& (\mathbf{E}+\mathbf{A}) \cdot \hat{\boldsymbol{\phi}}_{p_{\bullet}+4 ; 2\left(p_{\bullet}+1\right)}^{\prime}+\mathbf{A}_{p_{\bullet}+4 ; 2\left(p_{\bullet}+1\right)} \cdot \hat{\boldsymbol{\phi}}_{p_{\bullet}+4 ; 2\left(p_{\bullet}+1\right)}=\mathbf{R}_{2\left(p_{\bullet}+1\right) ; p_{\bullet}+1}, \\
& \mathbf{B} \cdot \hat{\boldsymbol{\phi}}_{p_{\bullet}+4 ; 2\left(p_{\bullet}+1\right)}^{\prime}+\mathbf{B}_{p_{\bullet}+4 ; 2\left(p_{\bullet}+1\right)} \cdot \hat{\boldsymbol{\phi}}_{p_{\bullet}+4 ; 2\left(p_{\bullet}+1\right)}=\mathbf{S}_{2\left(p_{\bullet}+1\right) ; p_{\bullet}+1},
\end{aligned}
$$

with $\mathbf{R}_{2(p \bullet+1) ; p_{\bullet}+1}$ and $\mathbf{S}_{2\left(p_{\bullet}+1\right) ; p_{\bullet}+1}$ as given in (26a)-26b). To conclude our argument, one needs to analyse the non-polynomial solutions to these equations. The detailed structure of the entries in the non-homogeneous terms $\mathbf{R}_{2(p \bullet+1) ; p \bullet+1}$ and $\mathbf{S}_{2\left(p_{\bullet}+1\right) ; p \bullet+1}$ can be obtained from the explicit calculations of [13. In particular, one has that these terms are polynomials of order $2 p_{\bullet}+10$ in $\tau$. More importantly, it can be explicitly verified that

$$
\begin{aligned}
& \partial_{\tau}^{8} \mathbf{R}_{2(p \bullet+1) ; p \bullet+1}=\tilde{\mathbf{R}}_{2(p \bullet+1) ; p \bullet+1}(\tau)(1-\tau)^{p \bullet-10}(1+\tau)^{p \bullet-10} \\
& \partial_{\tau}^{8} \mathbf{S}_{2(p \bullet+1) ; p_{\bullet}+1}=\tilde{\mathbf{S}}_{2(p \bullet+1) ; p \bullet+1}(\tau)(1-\tau)^{p \bullet-10}(1+\tau)^{p \bullet-10}
\end{aligned}
$$

where $\tilde{\mathbf{R}}_{2(p \bullet+1) ; p_{\bullet}+1}(\tau)$ and $\tilde{\mathbf{S}}_{2(p \bullet+1) ; p_{\bullet}+1}(\tau)$ have entries which are polynomials of degree 18 in $\tau$. Thus, the structure of the solutions to equations (31a)- 31b is best analysed if one takes 8 $\tau$-derivatives of the equations. From here arguments similar to those in [22] allow to show that

$$
\begin{aligned}
& \partial_{\tau}^{8} \hat{\phi}_{p_{\bullet}+4 ; 2\left(p_{\bullet}+1\right)}={\stackrel{\circ}{w_{2 ; 4}}}_{\breve{w}_{p_{\bullet}+1 ; 2(p \bullet+1)}}(1-\tau)^{p_{\bullet}-11}(1+\tau)^{p \bullet-11} \\
& \times\left(\hat{\boldsymbol{\varphi}}_{p_{\bullet}+4}(\tau)+\hat{\boldsymbol{\varphi}}_{p_{\bullet}+4}^{+}(\tau) \ln (1+\tau)+\hat{\boldsymbol{\varphi}}_{p_{\bullet}+4}^{-}(\tau) \ln (1-\tau)\right),
\end{aligned}
$$

with $\hat{\boldsymbol{\varphi}}_{p_{\bullet}+4}(\tau), \hat{\boldsymbol{\varphi}}_{p_{\bullet}+4}^{ \pm}(\tau)$ having entries which are polynomials of degree 24 in $\tau$.

Integrating the expressions (30) and (32) one finds that the polynomials multiplying the $\ln (1 \pm \tau)$ in $\widetilde{\phi}_{p_{\bullet}+4 ; 2\left(p_{\bullet}+1\right)}$ are of degree $2 p_{\bullet}+14$ and have an overall factor of $m^{3} \breve{w}_{p_{\bullet}+1 ; 2\left(p_{\bullet}+1\right)}$, whereas those in $\hat{\phi}_{p_{\bullet}+4 ; 2\left(p_{\bullet}+1\right)}$ are of degree $2 p_{\bullet}+10$ and have an overall factor $\stackrel{\circ}{2 ; 4}_{2,} \breve{w}_{p_{\bullet}+1 ; 2(p \bullet+1)}$. It follows that the polyhomogeneous terms in $\tilde{\phi}_{p_{\bullet}+4 ; 2\left(p_{\bullet}+1\right)}$ and $\hat{\phi}_{p_{\bullet}+4 ; 2\left(p_{\bullet}+1\right)}$ cannot cancel each other to produce a $\breve{\phi}_{p_{\bullet}+4 ; 2\left(p_{\bullet}+1\right)}$ which is entirely polynomial. Thus, one has the following:

Proposition 6. The solutions to the order $p_{\bullet}+4$ transport equations $(25 \mathrm{~b})-(25 \mathrm{c})$ with data given by equation (27) have no polynomial solutions unless $\breve{w}_{p_{\bullet}+1 ; 2\left(p_{\bullet}+1\right), k}=0$. If this condition is not satisfied the solutions develop logarithmic singularities at $\tau= \pm 1$ and the solutions are of class $C^{\omega}(-1,1) \cap C^{p \bullet+3}[-1,1]$.

\section{The main result}

The discussion in the previous sections is summarised in the following result.

Proposition 7. Given a time symmetric initial data set which in a neighbourhood $\mathcal{B}_{a}$ of infinity is static up to order $p=p_{\bullet}$ (in the sense of Definition 2), the solutions to the transport equations for the orders $p=p_{\bullet}+1, p_{\bullet}+2, p_{\bullet}+3$ are polynomial in $\tau$, and hence, extend smoothly through the critical sets $\mathcal{I}^{ \pm}$. On the other hand, the solutions at order $p=p_{\bullet}+4$ contain logarithmic singularities which can be avoided if an only if the initial data is, in fact, static up to order $p=p_{\bullet}+1$. 
As in the case of the analysis given in 22], one can use the previous result to implement an induction argument in which the explicit computer algebra calculations of [19] play the role of the base step to obtain the main result on this article.

Theorem 3. The solution to the regular finite initial value problem at spatial infinity for time

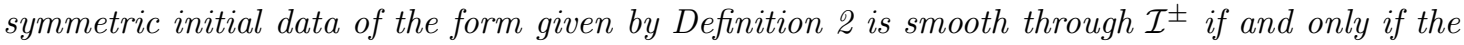
restriction of the data to $\mathcal{I}^{0}$ coincides with the restriction of static data at every order - that is, if their jets of order $p$ coincide for all p. Furthermore, the analyticity of the setting implies that the data is exactly static in a neighbourhood of infinity.

As already mentioned in the introduction, in order to complete the analysis of the solutions of the transport equations at the critical sets for general analytic time symmetric initial data sets, one needs (conformally invariant) conditions which reduce the initial data set to data with a static massless part in the sense discussed in Section 2, This problem will be analysed elsewhere.

\section{Acknowledgements}

This research was funded by an EPSRC Advanced Research Fellowship. I thank H. Friedrich, A. Aceña, T. Bäckdahl and C. Lübbe for useful discussions.

\section{References}

[1] A. Ashtekar, Lectures on non-perturbative canonical gravity, World Scientific, 1991.

[2] R. Beig, Conformal properties of static spacetimes, Class. Quantum Grav. 8, 263 (1991).

[3] D. R. Brill \& R. W. Lindquist, Interaction energy in geometrostatics, Phys. Rev. 131, 471 (1963).

[4] J. Frauendiener, Numerical treatment of the hyperboloidal initial value problem for the vacuum Einstein equations.I. The conformal field equations, Phys. Rev. D 58, 064002 (1998).

[5] H. Friedrich, On purely radiative space-times, Comm. Math. Phys. 103, 35 (1986).

[6] H. Friedrich, On static and radiative space-times, Comm. Math. Phys. 119, 51 (1988).

[7] H. Friedrich, Einstein equations and conformal structure: existence of anti-de Sitter-type space-times, J. Geom. Phys. 17, 125 (1995).

[8] H. Friedrich, Gravitational fields near space-like and null infinity, J. Geom. Phys. 24, 83 (1998).

[9] H. Friedrich, Conformal Einstein evolution, in The conformal structure of spacetime: Geometry, Analysis, Numerics, edited by J. Frauendiener \& H. Friedrich, Lecture Notes in Physics, page 1, Springer, 2002.

[10] H. Friedrich, Conformal geodesics on vacuum spacetimes, Comm. Math. Phys. 235, 513 (2003).

[11] H. Friedrich, Smoothness at null infinity and the structure of initial data, in 50 years of the Cauchy problem in general relativity, edited by P. T. Chruściel \& H. Friedrich, Birkhausser, 2004.

[12] H. Friedrich, Static vacuum solutions from convergent null data expansions at space-like infinity, Ann. Henri Poincaré 8, 817 (2007).

[13] H. Friedrich \& J. Kánnár, Bondi-type systems near space-like infinity and the calculation of the NP-constants, J. Math. Phys. 41, 2195 (2000).

[14] C. W. Misner, The method of images in geometrodynamics, Ann. Phys. 24, 102 (1963). 
[15] R. Penrose \& W. Rindler, Spinors and space-time. Volume 1. Two-spinor calculus and relativistic fields, Cambridge University Press, 1984.

[16] R. Penrose \& W. Rindler, Spinors and space-time. Volume 2. Spinor and twistor methods in space-time geometry, Cambridge University Press, 1986.

[17] P. Sommers, Space spinors, J. Math. Phys. 21, 2567 (1980).

[18] J. A. Valiente Kroon, Does asymptotic simplicity allow for radiation near spatial infinity?, Comm. Math. Phys. 251 (2004).

[19] J. A. Valiente Kroon, A new class of obstructions to the smoothness of null infinity, Comm. Math. Phys. 244, 133 (2004).

[20] J. A. Valiente Kroon, Time asymmetric spacetimes near null and spatial infinity. I. Expansions of developments of conformally flat data, Class. Quantum Grav. 23, 5457 (2004).

[21] J. A. Valiente Kroon, Time asymmetric spacetimes near null and spatial infinity. II. Expansions of developments of initial data sets with non-smooth conformal metrics, Class. Quantum Grav. 22, 1683 (2005).

[22] J. Valiente Kroon, A rigidity property of asymptoticaly simple spacetimes arising from conformally flat data, Comm. Math. Phys. 298, 673 (2010). 